\title{
I \\ THE HOCHSCHILD COHOMOLOGY RING OF THE SINGULAR COCHAIN ALGEBRA OF A SPACE
}

\author{
KATSUHIKO KURIBAYASHI
}

\begin{abstract}
We determine the algebra structure of the Hochschild cohomology of the singular cochain algebra with coefficients in a field on a space whose cohomology is a polynomial algebra. A spectral sequence calculation of the Hochschild cohomology is also described. In particular, when the underlying field is of characteristic two, we determine the associated bigraded BatalinVilkovisky algebra structure on the Hochschild cohomology of the singular cochain on a space whose cohomology is an exterior algebra.
\end{abstract}

\section{INTRODUCTION}

The objective of this paper is to compute the Hochschild cohomology ring of the singular cochain algebra on a simply-connected space, whose cohomology is either a polynomial algebra or an exterior algebra. The motivational topics are in string topology as well as in the classification problem of thick subcategories of the triangulated category associated with a singular cochain algebra on a space.

Let $M$ be a compact, oriented $d$-dimensional smooth manifold and $L M=$ $\operatorname{map}\left(S^{1}, M\right)$ the space of free loops on $M$. In [6], Chas and Sullivan have defined a product on the shifted homology $\mathbb{H}_{*}(L M):=H_{*+d}(L M)$, which is called the loop homology of $M$, and have shown that the homology, moreover, has the structure of a Batalin-Vilkovisky algebra. A result due to Cohen and Jones in 9] asserts that there exists an isomorphism of algebras between the loop homology of $M$ and the Hochschild cohomology ring of the singular cochain algebra on $M$; see also 8 . This allows one to describe the loop homology in terms of differential homological algebra. For various developments along this line, we refer the reader to [14, 15, 16, 17, 18, 26, 27, 33, 34, 35, 46, 45]. Especially, Menichi 34] has shown that the Hochschild cohomology of the singular cochain on a Poincaré duality space admits the structure of a Batalin-Vilkovisky algebra with the B-V operator given rise to by the Connes coboundary map. Thus we are also led to the study of the extended structure of the Hochschild cohomology.

As for global nature of singular cochains, Jørgensen has investigated the derived category of the singular cochain on a Poincaré duality space by applying AuslanderReiten theory. In particular, the Auslander-Reiten quiver of the full subcategory consisting of compact objects is determined in 24] and 25], see also [40. Such the result brings us to the study of topological spaces with categorical representation theory. Indeed, it is expected that numerical invariants, which appear and grow uniquely in the representation theory, capture topological properties of spaces via

2000 Mathematics Subject Classification: 16E40, 16E45, 55P35.

Key words and phrases. Hochschild cohomology, singular cochain algebra, Batalin-Vilkovisky algebra, Koszul-Tate resolution.

Department of Mathematical Sciences, Faculty of Science, Shinshu University, Matsumoto, Nagano 390-8621, Japan e-mail:kuri@math.shinshu-u.ac.jp 
functors from the category of spaces to an algebraic one. The author has introduced in [30. a new topological invariant, which is called the level of a space. For a space $M$ over a given space, the level of $M$ measures the number of steps to build the singular cochain on the space $M$ from that on a more fundamental space under an appropriate rule. We refer the reader to 31 for a linkage between the level and the Lusternik-Schnirelmann category of a space.

The origin of the numerical invariant level is in the study of the dimensions of triangulated categories; see for example [38. Then the level of an object in a triangulated category was first introduced by Avramov, Buchweitz, Iyengar and Miller in [1. It is also mentioned that, relying on knowledge of the levels of vertices on the Auslander-Reiten quiver alluded to above, the explicit calculation of the level for the total space of a bundle over the sphere is performed in [30].

Recently, Benson, Iyengar and Krause [3] have proved a classification theorem of thick subcategories of a triangulated category endowed with a ring homomorphism from a (graded) commutative ring to the graded center of the category; see Appendix for terminology. Let $C^{*}(X ; \mathbb{K})$ be the singular cochain algebra on a space $X$ with coefficients in a field $\mathbb{K}$ and $\mathrm{D}\left(C^{*}(X ; \mathbb{K})\right)$ the derived category of differential graded modules over the differential graded algebra $C^{*}(X ; \mathbb{K})$. Then the level of a space over $X$ is defined in the category $\mathrm{D}\left(C^{*}(X ; \mathbb{K})\right)$, which has the structure of a triangulated category. Therefore, we also expect that an explicit classification of thick subcategories of $\mathrm{D}\left(C^{*}(X ; \mathbb{K})\right)$ plays an important role in the study of the levels of spaces because the invariants are defined by filtering a suitable thick subcategory of $\mathrm{D}\left(C^{*}(X ; \mathbb{K})\right)$. In particular, one can take the Hochschild cohomology ring of $C^{*}(X ; \mathbb{K})$ as the graded commutative ring that may be the key to the classification of thick subcategories of the triangulated category $\mathrm{D}\left(C^{*}(X ; \mathbb{K})\right)$.

Thus the Hochschild cohomology of the singular cochain on a space becomes one of our great interests. However, there are few results on explicit calculations of the Hochschild cohomology rings of singular cochain algebras $C^{*}(X ; \mathbb{K})$ except for the case that the cohomology algebra $H^{*}(X ; \mathbb{K})$ is generated by a single element; see [33, 47.

As mentioned above, in this paper, we confine our attention mainly to the calculation of the Hochschild cohomology $H H^{*}\left(C^{*}(X ; \mathbb{K}) ; C^{*}(X ; \mathbb{K})\right)$ of the singular cochain on a simply-connected space $X$ whose cohomology is either a polynomial algebra or an exterior algebra. Unless otherwise explicitly stated, it is assumed that a space has the homotopy type of a CW-complex.

Our results in this paper are now described.

Theorem 1.1. Let $X$ be a simply-connected space whose mod $p$ cohomology is a polynomial algebra, say $H^{*}(X ; \mathbb{Z} / p) \cong \mathbb{Z} / p\left[x_{1}, x_{2}, \ldots, x_{n}\right]$. Then

$$
H H^{*}\left(C^{*}(X ; \mathbb{Z} / p) ; C^{*}(X ; \mathbb{Z} / p)\right) \cong \mathbb{Z} / p\left[x_{1}, x_{2}, \ldots, x_{n}\right] \otimes \wedge\left(u_{1}^{*}, u_{2}^{*}, \ldots, u_{n}^{*}\right)
$$

as an algebra, where $\operatorname{deg} u_{i}^{*}=-\operatorname{deg} x_{i}+1$.

Before describing another result on the computation of the Hochschild cohomology, we here recall the definition of the Batalin-Vilkovisky algebra.

Definition 1.2. A commutative graded algebra $A^{*}$ is a Batalin-Vilkovisky algebra if $A^{*}$ is equipped with an operation $\Delta: A^{*} \rightarrow A^{*-1}$ such that $\Delta^{2}=0$ and

$$
\begin{aligned}
\Delta(a b c)= & \Delta(a b) c+(-1)^{|a|} a \Delta(b c)+(-1)^{(|a|-1)|b|} b \Delta(a c) \\
& -(\Delta a) b c-(-1)^{|a|} a(\Delta b) c-(-1)^{|a|+|b|} a b(\Delta c) .
\end{aligned}
$$


The map $\Delta$ is called the $\mathrm{B}-\mathrm{V}$ operator.

Observe that the action of the B-V operator on the product of three elements is determined exactly by knowledge of that on the product of two elements.

Let $\mathbb{K}$ be a fixed field. A path-connected space $M$ is called a Poincaré duality space of formal dimension $m$ if the space $M$ is equipped with an orientation class $[M] \in H_{m}(M ; \mathbb{K})$ such that the cap product

$$
-\cap[M]: H^{*}(M ; \mathbb{K}) \rightarrow H_{m-*}(M ; \mathbb{K})
$$

is an isomorphism. The fundamental class of $M$ is the element $\omega_{M}$ such that $\left\langle\omega_{M},[M]\right\rangle=1$, where $\langle$,$\rangle denotes the Kronecker product.$

In what follows, for a space $X$, we may write $C^{*}(X)$ for $C^{*}(X ; \mathbb{K})$. Let $M$ be a simply-connected Poincaré duality space. Theorem 4.3 below states that the Moore spectral sequence (MSS for short) converges to $H H^{*}\left(C^{*}(M) ; C^{*}(M)\right)$ as a Batalin-Vilkovisky algebra. More precisely, each term of the MSS admits a differential Batalin-Vilkovisky algebra structure and the induced filtration on $H H^{*}\left(C^{*}(M) ; C^{*}(M)\right)$ respects the B-V operator defined by Menichi [34. Moreover, the $E_{\infty}$-term is isomorphic to the bigraded algebra $\operatorname{Gr} H H^{*}\left(C^{*}(M) ; C^{*}(M)\right)$ associated with the filtration as a bigraded Batalin-Vilkovisky algebra equipped with the B-V operator of bidegree $(-1,0)$.

The MSS with the differential Batalin-Vilkovisky algebra structure provides a new method for computing the Hochschild cohomology of the singular cochain on a space. In fact by applying the spectral sequence, we establish

Theorem 1.3. Let $M$ be a simply-connected space whose mod 2 cohomology is an exterior algebra, say $H^{*}(M ; \mathbb{Z} / 2) \cong \wedge\left(y_{1}, y_{2}, \ldots, y_{l}\right)$. Suppose further that the operation $S q^{1}$ vanishes on the cohomology. Then as a bigraded Batalin-Vilkovisky algebra,

$$
\operatorname{Gr} H H^{*}\left(C^{*}(M ; \mathbb{Z} / 2) ; C^{*}(M ; \mathbb{Z} / 2)\right) \cong \wedge\left(y_{1}, y_{2}, \ldots, y_{l}\right) \otimes \mathbb{Z} / 2\left[\nu_{1}^{*}, \nu_{2}^{*}, \ldots, \nu_{l}^{*}\right]
$$

in which $\Delta\left(y_{j}\right)=0, \Delta\left(\nu_{i}^{*}\right)=0, \Delta\left(y_{i} y_{j}\right)=0, \Delta\left(\nu_{i}^{*} \nu_{j}^{*}\right)=0$ for $1 \leq i, j \leq l$ and $\Delta\left(y_{i} \nu_{j}^{*}\right)=\delta_{i j} \cdot 1$, where bideg $y_{j}=\left(0, \operatorname{deg} y_{j}\right)$ and bideg $\nu_{j}^{*}=\left(1,-\operatorname{deg} y_{j}\right)$ for $1 \leq j \leq l$.

Observe that the space $M$ as in Theorem 1.3 is a Poincaré duality space with a orientation class $[M]$ which is the dual to a top non-zero element of the cohomology.

For a very special case, we can solve the extension problems on the product and on the B-V operator, which appear in the bigraded Batalin-Vilkovisky algebra $\operatorname{Gr} H H^{*}\left(C^{*}(M ; \mathbb{Z} / 2) ; C^{*}(M ; \mathbb{Z} / 2)\right)$; see Corollary 4.6. It seems that the result is the first computational example obtained by means of the MSS.

Remark 1.4. In general, the squaring operation $S q^{1}$ acts non-trivially on the mod 2 cohomology algebra of the Stiefel manifold of the form $M=S O(m+n) / S O(n)$ even if the algebra is an exterior algebra, see [36. However, thanks to the results [29, Corollary 5] and Proposition 3.2 below, we see that the conclusion of Theorem 1.3 remains valid for the Stiefel manifold $S O(m+n) / S O(n)$ provided $m \leq \min \{4, n\}$.

The rest of this paper is organized as follows. In Section 2, we recall the cup product of the Hochschild cohomology of a differential graded algebra and prove Theorem 1.1. Section 3 is devoted to proving the assertion in Theorem 1.3 concerning the bigraded algebra structure. To this end, we review the Moore spectral sequence converging to the Hochschild cohomology of the singular cochain algebra 
on a space. In Section 4, we discuss a Batalin-Vilkovisky algebra structure on the spectral sequence. Moreover fundamental properties of the Moore spectral sequence are considered here. In consequence, Theorem 1.3 is proved completely. Though Appendix has no result, we describe how one can take the loop homology into the categorical representation theory via the Hochschild cohomology.

\section{The Cup PROduct In The Hochschild COHOMOLOGY}

We begin with the definition of the Hochschild cochain complex. Let $(A, d)$ be an augmented differential graded algebra over a field $\mathbb{K}$ and $s \bar{A}$ denote the suspension of the augmentation ideal $\bar{A}$; that is, $(s \bar{A})^{n}=\bar{A}^{n+1}$. Let $T(s \bar{A})$ stand for the tensor algebra on $s \bar{A}$. The two-sided normalized bar complex $\mathbb{B}(A ; A ; A)$ is the tensor product $A \otimes T(s \bar{A}) \otimes A$ with the differential $d_{\mathbb{B}}=d_{1}+d_{2}$ defined by

$$
\begin{aligned}
d_{1}\left(a\left[a_{1}\left|a_{2}\right| \ldots \mid a_{k}\right] b\right)= & d(a)\left[a_{1}\left|a_{2}\right| \ldots \mid a_{k}\right] b-\sum_{i=1}^{k}(-1)^{\varepsilon_{i}} a\left[a_{1}\left|a_{2}\right| \ldots\left|d\left(a_{i}\right)\right| \ldots \mid a_{k}\right] b \\
& +(-1)^{\varepsilon_{k+1}} a\left[a_{1}\left|a_{2}\right| \ldots \mid a_{k}\right] d(b), \\
d_{2}\left(a\left[a_{1}\left|a_{2}\right| \ldots \mid a_{k}\right] b\right)= & (-1)^{|a|} a a_{1}\left[a_{2}|\ldots| a_{k}\right] b+\sum_{i=2}^{k}(-1)^{\varepsilon_{i}} a\left[a_{1}\left|a_{2}\right| \ldots\left|a_{i-1} a_{i}\right| \ldots \mid a_{k}\right] b \\
& -(-1)^{\varepsilon_{k}} a\left[a_{1}\left|a_{2}\right| \ldots \mid a_{k-1}\right] a_{k} b,
\end{aligned}
$$

where $\varepsilon_{i}=|a|+\sum_{j<i}\left(\left|s a_{j}\right|\right)$.

Let $\left(N, d_{N}\right)$ be a differential graded $A$-module. Then by definition the Hochschild cochain complex is a complex $\mathbf{C}(A, N)=\left\{\mathbf{C}^{n}(A, N), \partial\right\}$ of the form

$$
\mathbf{C}^{n}(A, N)=\operatorname{Hom}_{A \otimes A^{o p}}^{n}(\mathbb{B}(A ; A ; A), N)
$$

with the differential $\partial$ defined by $\partial(f)=d_{N} f-(-1)^{|f|} f d_{\mathbb{B}}$. The Hochschild cohomology $H H^{*}(A ; N)$ is defined to be the homology of the complex $\mathbf{C}(A, N)$. It follows from [13, Proposition 19.2] that the multiplication on $A$ induces a semi-free resolution $\mathbb{B}(A ; A ; A) \stackrel{\simeq}{\rightarrow} A$ of $A \otimes A^{o p}$-modules. This enables us to regard the Hochschild cohomology as the Ext-group $\operatorname{Ext}_{A \otimes A^{o p}}(A, N)$ in the sense of Moore; see [10, Appendix].

For a vector space $V$, we denote by $V^{\vee}$ the dual vector space to $V$ unless otherwise noted. For a basis $\left\{v_{i}\right\}_{i \in I}$ for $V$, the dual basis is denoted by $\left\{v_{i}^{*}\right\}_{i \in I}$.

We here recall a Koszul-Tate resolution of a graded commutative algebra. Let $\Lambda$ a graded algebra over a field $\mathbb{K}$ of the form

$$
\Lambda=\wedge\left(y_{1}, \ldots, y_{l}\right) \otimes \mathbb{K}\left[x_{1}, \ldots, x_{n}\right] /\left(\rho_{1}, \ldots, \rho_{m}\right),
$$

where $\rho_{1}, \ldots, \rho_{m}$ is a regular sequence in the polynomial algebra $\mathbb{K}\left[x_{1}, \ldots, x_{n}\right]$. We assume that each $\rho_{i}$ is decomposable. Observe that $\operatorname{deg} x_{i}$ is even and $\operatorname{deg} y_{i}$ is odd if the characteristic of $\mathbb{K}$ is greater than two. The algebra $\Lambda$ is called a graded complete intersection algebra.

Proposition 2.1. ([43, Proposition 3.5] [29, Proposition 1.1]) Under the above hypothesis, there exists a projective resolution $\mathcal{F} \stackrel{\varphi}{\rightarrow} \Lambda \rightarrow 0$ of $\Lambda$ as a left $\Lambda \otimes \Lambda^{o p}$. module such that

$$
\mathcal{F}=\Lambda \otimes \Lambda \otimes \Gamma\left[\nu_{1}, \ldots, \nu_{l}\right] \otimes \wedge\left(u_{1}, \ldots, u_{n}\right) \otimes \Gamma\left[w_{1}, \ldots, w_{m}\right],
$$

$d(\Lambda \otimes \Lambda)=0, d\left(\nu_{i}\right)=y_{i} \otimes 1-1 \otimes y_{i}, d\left(u_{j}\right)=x_{j} \otimes 1-1 \otimes x_{j}, d\left(\gamma_{r}\left(w_{i}\right)\right)=$ $\left(\sum_{j=1}^{n} \zeta_{i j} u_{j}\right) \otimes \gamma_{r-1}\left(w_{i}\right)$ and $\varphi$ is the multiplication of $\Lambda$, where bideg $\lambda=(0, \operatorname{deg} \lambda)$ 
for $\lambda \in \Lambda \otimes \Lambda$, bideg $\nu_{i}=\left(-1, \operatorname{deg} y_{i}\right)$, bideg $u_{j}=\left(-1, \operatorname{deg} x_{i}\right)$ and bideg $w_{i}=$ $\left(-2, \operatorname{deg} \rho_{i}\right)$. Here $\zeta_{i j}$ is an appropriate element of $\mathbb{K}\left[x_{1}, \ldots, x_{n}\right] \otimes \mathbb{K}\left[x_{1}, \ldots, x_{n}\right]$ which satisfies the condition that

$$
\rho_{i} \otimes 1-1 \otimes \rho_{i}=\sum_{j=1}^{n} \zeta_{i j}\left(x_{j} \otimes 1-1 \otimes x_{j}\right) \quad \text { and } \quad \varphi\left(\zeta_{i j}\right)=\frac{\partial \rho_{i}}{\partial x_{j}} .
$$

It is readily seen that $\mathcal{F}$ is semi-free and hence $\varphi: \mathcal{F} \rightarrow \Lambda$ in Proposition 2.1 is a semi-free resolution of $\Lambda$. In what follows, we shall call the resolution the Koszul-Tate resolution of $\Lambda$.

Remark 2.2. In the case where the algebra $\Lambda$ is a truncated polynomial algebra generated by a single element, the Koszul-Tate resolution is nothing but the periodic resolution, which is used in [47, 22, 7] to compute the Hochschild (co)homology of $\Lambda$. See also 21] for the Hochschild cohomology ring of an exterior algebra.

Let $A$ be an augmented differential graded algebra (DG algebra), $M$ and $N$ differential graded bimodules over $A$. Let $P \stackrel{\simeq}{\rightrightarrows} A$ be a semi-free resolution of $A$ as a $A \otimes A^{o p}$-module. Then the cup product

$$
\smile: H H^{*}(A ; M) \otimes H H^{*}(A ; N) \rightarrow H H^{*}\left(A, M \otimes_{A} N\right)
$$

is defined with an $A \otimes A^{o p}$-chain map $D: P \rightarrow P \otimes_{A} P$, which is a lift of the identity map on $A$, by the composite

$$
f \smile g: P \stackrel{D}{\rightarrow} P \otimes_{A} P \stackrel{f \otimes g}{\rightarrow} N \otimes_{A} N
$$

for $f, g \in \operatorname{Hom}_{A \otimes A^{o p}}(A, N)$. We call the map $D: P \rightarrow P \otimes_{A} P$ a diagonal map.

Suppose that there exists an $A \otimes A^{o p}$-module map $N \otimes_{A} N \rightarrow N$. Then the cup product on the Hochschild cochain complex $\mathbf{C}(A ; N)$ makes the Hochschild cohomology $H H^{*}(A ; N)$ into a graded algebra. Observe that, in the case $M=N=$ $A$, the algebra $H H^{*}(A ; A)$ is graded commutative; see for example 39, Proposition 1.2]. Moreover, using the $A \otimes A^{o p}$-module isomorphism $A \otimes_{A} N \stackrel{\cong}{\rightrightarrows} N$, which is induced by the right $A$-module structure on $N$, we give $H H^{*}(A ; N)$ an $H H^{*}(A ; N)$ module structure

$$
\smile: H H^{*}(A ; A) \otimes H H^{*}(A ; N) \rightarrow H H^{*}(A, N) .
$$

We here look at a bigraded algebra structure on the Hochschild cohomology $H H^{*}(A ; N)$ provided the differentials on $A$ and $N$ are trivial. Let $\mathbf{C}^{p, q}$ be the subspace $\operatorname{Hom}_{A \otimes A^{o p}}^{p+q}\left(P_{-p}, N\right)$ of $\mathbf{C}(A ; N)$. Then it follows that the differential $d$ of $\mathbf{C}(A ; N)$ maps $\mathbf{C}^{p, q}$ into $\mathbf{C}^{p+1, q}$. This implies that $\left\{\mathbf{C}^{*, q}, d\right\}_{p \geq 0}$ is a subcomplex of the Hochschild cochain complex $\mathbf{C}(A ; N)$ for any $q$ and hence we have

$$
H H^{n}(A ; N)=\bigoplus_{p+q=n} H H^{p, q}(A, N)
$$

where $H H^{*, q}(A, N)$ denotes the homology of the complex $\left\{\mathbf{C}^{*, q}, d\right\}_{p \geq 0}$. Let $D$ : $P \rightarrow P \otimes_{A} P$ be a diagonal map. Then by definition one sees that the image $D\left(P_{-p}\right)$ is included in $\oplus_{i+j=-p} P_{i} \otimes_{A} P_{j}$. This yields that the Hochschild cohomology $H H^{n}(A ; N)$ admits a bigraded algebra structure; that is,

$$
\smile: H H^{p, q}(A, N) \otimes H H^{p^{\prime}, q^{\prime}}(A, N) \rightarrow H H^{p+p^{\prime}, q+q^{\prime}}(A, N) .
$$

The uniqueness of the cup product on the Hochschild homology; see [39, §1] [41, $\S 2]$, allows us to define the product with a tractable diagonal map. We construct 
an explicit diagonal map for the Koszul-Tate resolution of a DG algebra, which is the tensor product of a polynomial algebra and an exterior algebra with the trivial differential.

Suppose that $\Lambda$ is an algebra of the form $\wedge\left(y_{1}, \ldots, y_{l}\right) \otimes \mathbb{K}\left[x_{1}, \ldots, x_{n}\right]$. We write $\Lambda \otimes$ $\Lambda \otimes \mathcal{E}$ for the graded algebra $\mathcal{F}$ mentioned in Proposition 2.1 with $\mathcal{E}=\Gamma\left[\nu_{1}, \ldots, \nu_{l}\right] \otimes$ $\Lambda\left(u, \ldots, u_{n}\right)$. Define a $\Lambda \otimes \Lambda^{o p}$-homomorphism

$$
D: \Lambda \otimes \Lambda \otimes \mathcal{E} \rightarrow \Lambda \otimes \Lambda \otimes \mathcal{E} \otimes_{\Lambda} \Lambda \otimes \Lambda \otimes \mathcal{E}
$$

on the generators of the algebra $\mathcal{F}=\Lambda \otimes \Lambda \otimes \mathcal{E}$ by

$$
\begin{gathered}
D\left(u_{j}\right)=1 \otimes 1 \otimes u_{j} \otimes_{\Lambda} 1 \otimes 1 \otimes 1+1 \otimes 1 \otimes 1 \otimes_{\Lambda} 1 \otimes 1 \otimes u_{j}, \\
D\left(\gamma_{k}\left(\nu_{i}\right)\right)=\sum_{s+t=k} 1 \otimes 1 \otimes \gamma_{s}\left(\nu_{i}\right) \otimes_{\Lambda} 1 \otimes 1 \otimes \gamma_{t}\left(\nu_{i}\right)
\end{gathered}
$$

and extend them to the whole module with

$$
D\left(\alpha \gamma_{i_{1}}\left(\nu_{1}\right) \cdots \gamma_{i_{l}}\left(\nu_{l}\right) u_{1}^{\varepsilon_{1}} \cdots u_{n}^{\varepsilon_{n}}\right)=\alpha D\left(\gamma_{i_{1}}\left(\nu_{1}\right)\right) \cdots D\left(\gamma_{i_{l}}\left(\nu_{l}\right)\right) D\left(u_{1}\right)^{\varepsilon_{1}} \cdots D\left(u_{n}\right)^{\varepsilon_{n}},
$$

where $\varepsilon_{i}=0$ or 1 and $\alpha \in \Lambda \otimes \Lambda^{o p}$. Observe that

$\lambda_{1} \otimes \lambda_{2}\left(\lambda \otimes \mu \otimes a \otimes_{\Lambda} \lambda^{\prime} \otimes \mu^{\prime} \otimes b\right)=(-1)^{\left|\lambda_{2}\right|\left(|\lambda|+|\mu|+|a|+\left|\lambda^{\prime}\right|\right)} \lambda_{1} \lambda \otimes \mu \otimes a \otimes_{\Lambda} \lambda^{\prime} \otimes \lambda_{2} \mu^{\prime} \otimes b$,

for $\lambda_{1} \otimes \lambda_{2} \in \Lambda \otimes \Lambda^{o p}$ and $\lambda \otimes \mu \otimes a \otimes_{\Lambda} \lambda^{\prime} \otimes \mu^{\prime} \otimes b \in \mathcal{F} \otimes_{\Lambda} \mathcal{F}$.

Lemma 2.3. The $\Lambda \otimes \Lambda^{o p}$-homomorphism $D$ is a diagonal map.

Proof. The differential $d$ is a derivation on the algebra $\mathcal{F}$ and hence so is $\partial:=$ $d \otimes 1+1 \otimes d$ on $\mathcal{F} \otimes_{\Lambda} \mathcal{F}$. Thus in order to prove the lemma, it suffices to show that

$$
\partial D(v)=D d(v)
$$

for any $v \in\left\{\gamma_{i_{1}}\left(\nu_{1}\right), \ldots, \gamma_{i_{l}}\left(\nu_{l}\right) \mid i_{t} \geq 1\right\} \cup\left\{u_{1}, \ldots, u_{n}, x_{i}\right\}$. In fact, we choose a base $x$ for $\mathcal{F}$ of the form $\alpha v_{i} \cdots v_{l} \cdot v_{l+1} \cdots v_{l+k}$, where $\alpha \in \Lambda \otimes \Lambda^{o p}, v_{j}=\gamma_{i_{j}}\left(\nu_{j}\right)$, $v_{l+i} \in\left\{u_{1}, \ldots, u_{n}\right\}$ and $v_{l+i} \neq v_{l+j}$ if $i \neq j$. Then we see that

$$
\begin{aligned}
\partial D(x) & =\partial\left(\alpha D\left(v_{1}\right) \cdots D\left(v_{l+k}\right)\right) \\
& =\alpha \sum_{1 \leq i \leq l+k} \pm D\left(v_{1}\right) \cdots \partial D\left(v_{i}\right) \cdots D\left(v_{l+k}\right) \\
& =\alpha \sum_{1 \leq i \leq l+k} \pm D\left(v_{1}\right) \cdots D\left(d v_{i}\right) \cdots D\left(v_{l+k}\right) \\
& =D\left(\alpha \sum_{1 \leq i \leq l+k} \pm v_{1} \cdots d v_{i} \cdots v_{l+k}\right)=D d(x) .
\end{aligned}
$$

Here \pm denotes the Koszul sign. The differential $d$ is closed under the subalgebras $\Lambda \otimes \Lambda \otimes \Gamma\left[\nu_{i}\right]$ and $\Lambda \otimes \Lambda \otimes \wedge\left(u_{j}\right)$ for $i=1, . ., l$ and $j=1, \ldots, n$ so that the forth equality follows from the definition of the diagonal map $D$. We now verify the 
equality (2.3). It follows that

$$
\begin{aligned}
& \partial D\left(\gamma_{k}\left(\nu_{i}\right)\right)=\partial\left(\sum_{s+t=k} 1 \otimes 1 \otimes \gamma_{s}\left(\nu_{i}\right) \otimes_{\Lambda} 1 \otimes 1 \otimes \gamma_{t}\left(\nu_{i}\right)\right) \\
= & \sum_{s+t=k}\left(y_{i} \otimes 1 \otimes \gamma_{s-1}\left(\nu_{i}\right) \otimes_{\Lambda} 1 \otimes 1 \otimes \gamma_{t}\left(\nu_{i}\right)-1 \otimes y_{i} \otimes \gamma_{s-1}\left(\nu_{i}\right) \otimes_{\Lambda} \otimes 1 \otimes \gamma_{t}\left(\nu_{i}\right)\right. \\
& \left.+1 \otimes 1 \otimes \gamma_{s}\left(\nu_{i}\right) \otimes_{\Lambda} y_{i} \otimes 1 \otimes \gamma_{t-1}\left(\nu_{i}\right)-1 \otimes 1 \otimes \gamma_{s}\left(\nu_{i}\right) \otimes_{\Lambda} 1 \otimes y_{i} \otimes \gamma_{t-1}\left(\nu_{i}\right)\right) \\
= & \sum_{s+t=k-1}\left(y_{i} \otimes 1 \otimes \gamma_{s}\left(\nu_{i}\right) \otimes_{\Lambda} 1 \otimes 1 \otimes \gamma_{t}\left(\nu_{i}\right)-1 \otimes 1 \otimes \gamma_{s}\left(\nu_{i}\right) \otimes_{\Lambda} 1 \otimes y_{i} \otimes \gamma_{t}\left(\nu_{i}\right)\right) \\
= & \left(y_{i} \otimes 1-1 \otimes y_{i}\right) \cdot\left(\sum_{s+t=k-1} 1 \otimes 1 \otimes \gamma_{s}\left(\nu_{i}\right) \otimes_{\Lambda} 1 \otimes 1 \otimes \gamma_{t}\left(\nu_{i}\right)\right) \\
= & D\left(\left(y_{i} \otimes 1-1 \otimes y_{i}\right) \gamma_{k-1}\left(\nu_{i}\right)\right)=\operatorname{Dd}\left(\gamma_{k}\left(\nu_{i}\right)\right)
\end{aligned}
$$

The same calculation as above enables us to conclude that $\partial D\left(u_{j}\right)=D d\left(u_{j}\right)$. We have the result.

Let $A$ be a $\Lambda$-bimodule equipped with a $\Lambda \otimes \Lambda^{o p}$-module map $A \otimes_{\Lambda} A \rightarrow A$. In particular, if $A$ is a commutative graded algebra over $\Lambda$, then $A$ is viewed as a $\Lambda$-bimodule with $\left(\lambda_{1} a\right) \lambda_{2}=\lambda_{1}\left(a \lambda_{2}\right):=(-1)^{|a|\left|\lambda_{2}\right|} \lambda_{1}\left(\lambda_{2} a\right)$ for $\lambda_{1}, \lambda_{2} \in \Lambda$ and $a \in A$. Moreover, a $\Lambda \otimes \Lambda^{o p}$-module map $A \otimes_{\Lambda} A \rightarrow A$ is naturally induced by the product on $A$.

Assume that $\Lambda$ is isomorphic to either a polynomial algebra $\mathbb{K}\left[x_{1}, \ldots, x_{n}\right]$ or an exterior algebra $\wedge\left(y_{1}, \ldots, y_{l}\right)$ and that $A$ is a commutative graded algebra over $\Lambda$ such that $\operatorname{dim} A<\infty$ if $\Lambda$ is an exterior algebra. We consider the DG algebra structure of the complex $\operatorname{Hom}_{\Lambda \otimes \Lambda}(\mathcal{F}, A)$, which computes the $E_{2}$-term of the spectral sequence introduced in the next section.

By assumption, one of algebras $A$ and $\mathcal{E}$ is of finite dimension. This allows us to obtain an isomorphism

$$
\theta: A \otimes \mathcal{E}^{\vee}=A \otimes \operatorname{Hom}_{\mathbb{K}}(\mathcal{E}, \mathbb{K}) \stackrel{\cong}{\rightrightarrows} \operatorname{Hom}_{\Lambda \otimes \Lambda}(\Lambda \otimes \Lambda \otimes \mathcal{E}, A)
$$

defined by $\theta(a \otimes f)(\lambda \otimes \alpha)=(-1)^{|\lambda|(|f|+|a|)} \lambda \cdot a f(\alpha)$, where $a \in A, \lambda \in \Lambda \otimes \Lambda, f \in \mathcal{E}^{\vee}$, $\alpha \in \mathcal{E}$ and · stands for the $\Lambda \otimes \Lambda$-module structure on $A$. Thus it follows that the vector space $A \otimes \mathcal{E}^{\vee}$ admits a differential algebra structure via the isomorphism $\theta$. As for the algebra structure, we see that for the dual base $\gamma_{k}\left(\nu_{i}\right)^{*}$ to $\gamma_{k}\left(\nu_{i}\right)$ and the dual base $u_{j}^{*}$ to $u_{j}$,

$$
a \otimes \gamma_{k}\left(\nu_{i}\right)^{*} \cdot b \otimes \gamma_{l}\left(\nu_{i}\right)^{*}=a b \otimes \gamma_{k+l}\left(\nu_{i}\right)^{*} \text { and } a \otimes u_{j}^{*} \cdot b \otimes u_{j}^{*}=0 .
$$

Observe that the total degree of the element $\gamma_{k}\left(\nu_{i}\right)^{*}$ is even if $p$ is odd. For any element $x$ in $\Lambda \otimes \Lambda \otimes \mathcal{E}$, each term of $d x$ has an element of the form $\lambda \otimes 1-1 \otimes \lambda$, where $\lambda \in \Lambda$; see Proposition 2.1. Moreover, since $(\lambda \otimes 1-1 \otimes \lambda) \cdot a=\lambda(1 a)-1(\lambda a)=0$ for $\lambda \in \Lambda$ and $a \in A$, it follows that for any $\Lambda \otimes \Lambda$-module map $\varphi: \Lambda \otimes \Lambda \otimes \mathcal{E} \rightarrow A$, $(d \varphi)(x)=(-1)^{|\varphi|} \varphi(d x)=0$ and hence the differential on $A \otimes \mathcal{E}^{\vee}$ is trivial. Thus we have

Proposition 2.4. As a bigraded algebra,

$$
H H^{*}(\Lambda ; A) \cong \begin{cases}A \otimes \mathbb{K}\left[\nu_{1}^{*}, \ldots, \nu_{l}^{*}\right] & \text { if } \Lambda=\wedge\left(y_{1}, . ., y_{l}\right), \\ A \otimes \wedge\left(u_{1}^{*}, \ldots, u_{n}^{*}\right) & \text { if } \Lambda=\mathbb{K}\left[x_{1}, . ., x_{n}\right],\end{cases}
$$

where bideg $a=(0, \operatorname{deg} a)$, bideg $\nu_{i}^{*}=\left(1,-\operatorname{deg} y_{i}\right)$ and $\operatorname{bideg} u_{j}^{*}=\left(1,-\operatorname{deg} x_{j}\right)$. 
Proof of Theorem 1.1. The proof of [37, 7.1 Theorem] implies that $C^{*}(X)$ is $\mathbb{K}$ formal; that is, there exists a sequence of quasi-isomorphisms which connects $C^{*}(X)$ with $H^{*}(X ; \mathbb{K})$. It follows from [16, 3.4 Proposition] that the Hochschild cohomology ring $H H\left(C^{*}(X) ; C^{*}(X)\right)$ is isomorphic to $H H\left(H^{*}(X) ; H^{*}(X)\right)$ as an algebra. Proposition 2.4 yields the result.

Remark 2.5. Let $\Lambda$ be a graded complete intersection algebra. The algebra structure of the Hochschild cohomology $H H^{*}(\Lambda, \Lambda)$ may be described in terms of cycles on the Koszul-Tate resolution in Proposition 2.1 if one has an explicit form of a diagonal map.

\section{A spectral sequence converging to the Hochschild COHOMOlogy RING OF A DG ALGEBRA}

For a space $X$, we assume that the cohomology $H^{*}(X ; \mathbb{K})$ is of finite type; that is, $\operatorname{dim} H^{i}(X ; \mathbb{K})<\infty$ for any $i$. Let $M$ and $N$ be connected spaces and $f: N \rightarrow M$ a map. The singular cochain algebra $C^{*}(N)$ is regarded as a $C^{*}(M)$-bimodule via the map $f^{*}: C^{*}(M) \rightarrow C^{*}(N)$ induced by $f$. Then it follows that the cup product gives rise to a $C^{*}(M) \otimes C^{*}(M)^{o p}$-module map $C^{*}(N) \otimes_{C^{*}(M)} C^{*}(N) \rightarrow C^{*}(N)$.

Theorem 3.1. (cf. [17, 1 Proposition] ) Under the above hypothesis, we assume further that $H^{*}(N)$ is of finite dimension. Then there exists a right-half plane cohomological spectral sequence $\left\{E_{r}^{*, *}, d_{r}\right\}$ converging to the Hochschild cohomology $H H^{*}\left(C^{*}(M) ; C^{*}(N)\right)$ as an algebra such that

$$
E_{2}^{p, q} \cong H H^{p, q}\left(H^{*}(M) ; H^{*}(N)\right)
$$

as a bigraded algebra.

Proof. Let $S$ be a complement of the vector subspace generated by cycles of $C^{d}(N)$, where $d=\sup \left\{n \mid H^{*}(N) \neq 0\right\}$. We define $I$ to be the two-sided ideal generated by $C^{>d}(N) \oplus S$. Then the projection $C^{*}(N) \rightarrow C^{*}(N) / I$ is a quasi-isomorphism of $A$-bimodules.

Let $A:=T V \stackrel{\simeq}{\rightarrow} C^{*}(M)$ be a TV model for the space $M$ in the sense of Halperin and Lemaire 20]. Let $\mathbb{B}_{*}(A ; A ; A)$ be the normalized bar complex mentioned in the previous section. We then define a decreasing filtration $\left\{F^{p} \mathbf{C}^{*}\right\}_{p \geq 0}$ of the Hochschild cochain complex $\mathbf{C}^{*}=\left\{\operatorname{Hom}_{A \otimes A^{o p}}\left(\mathbb{B}_{*}(A ; A ; A), C^{*}(N) / I\right)\right\}_{n \in \mathbb{Z}}$ by

$$
F^{p} \mathbf{C}^{n}=\prod_{s \geq p} \operatorname{Hom}_{A \otimes A^{o p}}^{n}\left(\mathbb{B}_{s}(A ; A ; A), C^{*}(N) / I\right),
$$

where $\mathbb{B}_{s}(A ; A ; A)=A \otimes s \bar{A}^{\otimes s} \otimes A$. Since $s \bar{A}$ has no element of degree zero, it follows that the number $\inf \left\{m \mid\left(\mathbb{B}_{s}(A ; A ; A)\right)^{m} \neq 0\right\}$ increases strictly if so does $s$. It is immediate that $\left(C^{*}(N) / I\right)^{<0}=0$ and $\left(C^{*}(N) / I\right)^{>d}=0$. These facts imply that the filtration $\left\{F^{p} \mathbf{C}^{*}\right\}_{p \geq 0}$ is bounded; that is, for any $n$, there exists $p(n)$ such that $F^{p} \mathbf{C}^{n}=0$ for $p>p(n)$.

Observe that $F^{0} \mathbf{C}^{*}=\mathbf{C}^{*}$ and that the cup product on the Hochschild cochain complex respects the filtration; that is, $F^{s} \mathbf{C}^{n} \smile F^{t} \mathbf{C}^{m} \subset F^{s+t} \mathbf{C}^{n+m}$. Therefore as usual we can construct a spectral sequence $\left\{E_{r}, d_{r}\right\}$, whose each term admits the structure of a differential graded algebra, by using the filtration. Since the filtration is bounded, it follows that the spectral sequence converses to $H H^{*}\left(C^{*}(M) ; C^{*}(N)\right)$ 
as an algebra. Moreover the Künneth theorem yields that the $E_{1}$-term is a complex of the form

$$
E_{1}^{p, q}=\operatorname{Hom}_{A \otimes A^{o p}}^{p+q}\left(\mathbb{B}_{p}\left(H^{*}(M) ; H^{*}(M) ; H^{*}(M)\right), H^{*}(N)\right)
$$

which is the Hochschild cochain complex. We have the result.

The spectral sequence in Theorem 3.1] is called the Moore spectral sequence.

Let $\mathbf{C}^{*}$ be the Hochschild complex mentioned in the proof of Theorem 3.1. Then the inclusion $i: F^{p} \mathbf{C}^{n} \rightarrow \mathbf{C}^{n}$ defines the submodule

$$
F^{p} H H^{n}:=\operatorname{Im}\left\{H(i): H^{n}\left(F^{p} \mathbf{C}^{*}\right) \rightarrow H H^{n}\left(C^{*}(M) ; C^{*}(N)\right)\right\}
$$

of the Hochschild cohomology $H H^{n}\left(C^{*}(M) ; C^{*}(N)\right)$. We define the associated bigraded module of $H H^{n}\left(C^{*}(M) ; C^{*}(N)\right)$ by

$$
\mathrm{Gr}^{p, q} H H^{*}\left(C^{*}(M) ; C^{*}(N)\right)=F^{p} H H^{p+q} / F^{p+1} H H^{p+q} .
$$

Observe that $\mathrm{Gr}^{p, q} H H^{*}\left(C^{*}(M) ; C^{*}(N)\right)$ is isomorphic to the vector space $E_{\infty}^{p, q}$ in the $E_{\infty}$-term of the Moore spectral sequence.

We prove a key proposition to proving the assertion in Theorem 1.3 concerning the bigraded algebra structure.

Proposition 3.2. Let $M$ be a simply-connected Poincaré duality space and $\left\{\widetilde{E}_{r}^{*, *}, \widetilde{d}_{r}\right\}$ the Eilenberg-Moore spectral sequence associated with the pull-back diagram

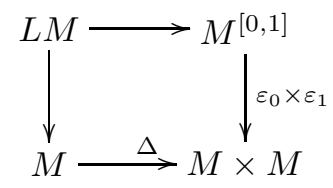

converging to the cohomology $H^{*}(L M ; \mathbb{K})$, where $\varepsilon_{i}$ denotes the evaluation map at $i$ for $i=0,1$ and $\Delta$ is the diagonal map. Let $\left\{E_{r}, d_{r}\right\}$ be the Moore spectral sequence converging to $\mathrm{HH}^{*}\left(C^{*}(M) ; C^{*}(M)\right)$. Then all the elements in the $E_{2}$ term of $\left\{\widetilde{E}_{r}^{*, *}, \widetilde{d}_{r}\right\}$ with total degree less than or equal to $l$ are permanent cycles if and only if so are all the elements in the $E_{2}$-term of $\left\{E_{r}, d_{r}\right\}$ with total degree greater than or equal to $-l+d-1$. In particular, the spectral sequence $\left\{\widetilde{E}_{r}^{*, *}, \widetilde{d}_{r}\right\}$ collapses at the $E_{2}$-term if and only if so does the Moore spectral sequence $\left\{E_{r}, d_{r}\right\}$.

Proof. Let $\left\{\mathbb{B}_{p}, d\right\}_{p \geq 0}$ be the normalized bar complex of $A:=H^{*}(M)$. The $E_{1^{-}}$ term of the spectral sequence $\left\{\widetilde{E}_{r}^{*, *}, \widetilde{d}_{r}\right\}$ is given by $\widetilde{E}_{1}^{-p, q}=\left(\mathbb{B}_{p} \otimes_{A \otimes A^{o p}} A\right)^{-p+q}$. Thus we see that the spectral sequence $\left\{\left(\widetilde{E}_{r}^{*, *}\right)^{\vee}, \widetilde{d}_{r}^{\vee}\right\}$, which is dual to $\left\{\widetilde{E}_{r}^{*, *}, \widetilde{d}_{r}\right\}$, converges to $H H^{*}\left(C^{*}(M) ; C^{*}(M)^{\vee}\right)$ and that

$$
\left(\widetilde{E}_{1}^{-p, q}\right)^{\vee}=\operatorname{Hom}^{p-q}\left(\mathbb{B}_{p} \otimes_{A \otimes A^{o p}} A, \mathbb{K}\right) \cong \operatorname{Hom}_{A \otimes A^{o p}}^{p-q}\left(\mathbb{B}_{p}, A^{\vee}\right)
$$

as complexes. The main theorem in [23] asserts that as a vector space $H_{-p+q}(L M) \cong$ $H H^{p-q}\left(C^{*}(M), C^{*}(M)^{\vee}\right)$.

Moreover, since $A$ is a commutative, it follows that the Poincaré duality gives an isomorphism $A \cong A^{\vee}$ of $A$-bimodules. Then we have an isomorphism

$$
\begin{aligned}
\left(\widetilde{E}_{2}^{-p, q}\right)^{\vee}=H\left(\widetilde{E}_{1}^{-p, q}\right)^{\vee} & =H\left(\operatorname{Hom}_{A \otimes A^{o p}}^{p-q}\left(\mathbb{B}_{p}, A^{\vee}\right)\right) \\
& \cong H\left(\operatorname{Hom}_{A \otimes A^{o p}}^{p-q+d}\left(\mathbb{B}_{p}, A\right)\right)=H\left(E_{1}^{p,-q+d}\right)=E_{2}^{p,-q+d} .
\end{aligned}
$$


The result [17, 13 Theorem] due to Félix, Thomas and Vigué-Poirrier allows us to obtain an isomorphism $H H^{*}\left(C^{*}(M) ; C^{*}(M)^{\vee}\right) \cong H H^{*+d}\left(C^{*}(M) ; C^{*}(M)\right)$; see also [34, Theorem 20]. Thus it turns out that the following conditions are equivalent: (1) all the elements in the $E_{2}$-term of $\left\{\widetilde{E}_{r}^{*, *}, \widetilde{d}_{r}\right\}$ with total degree less than or equal to $l$ are permanent cycles.

(2) $H^{n}(L M) \cong \bigoplus_{-p+q=n} \widetilde{E}_{2}^{-p, q}$ for any $n \leq l$.

(3) $H_{n}(L M) \cong \bigoplus_{p} H^{-n}\left(\operatorname{Hom}_{A \otimes A^{o p}}\left(\mathbb{B}_{p}, A^{\vee}\right)\right)$ for any $n \leq l$.

(4) $H H^{-n+d}\left(C^{*}(M) ; C^{*}(M)\right) \cong H H^{-n}\left(C^{*}(M) ; C^{*}(M)^{\vee}\right) \cong \bigoplus_{p-q=-n} E_{2}^{p,-q+d}$ for any $n \leq l$.

(5) all the elements in the $E_{2}$-term of $\left\{E_{r}, d_{r}\right\}$ with total degree greater than or equal to $-l+d-1$ are permanent cycles.

In fact, the isomorphisms mentioned above allow us to conclude that the conditions (2), (3) and (4) are equivalent. Since the vector space $E_{r+1}^{p, q}$ is a subquotient of $E_{r}^{p, q}$, more precisely,

$$
E_{r+1}^{p, q} \cong \operatorname{Ker}\left\{d_{r}: E_{r}^{p, q} \rightarrow E_{r}^{p+r, q+1-r}\right\} / \operatorname{Im}\left\{d_{r}: E_{r}^{p-r, q-1+r} \rightarrow E_{r}^{p, q}\right\},
$$

it follows that $\operatorname{dim} E_{r+1}^{p, q} \leq \operatorname{dim} E_{r}^{p, q}$. Then the equality holds if and only if all the elements in $E_{r}^{p, q}$ and $E_{r}^{p-r, q-1+r}$ are cycles. This yields that $\operatorname{dim} E_{\infty}^{p, q}=\operatorname{dim} E_{2}^{p, q}$ for $p+q \leq l$ if and only if the all the elements in $E_{2}^{p, q}$ are permanent cycles for $p+q \leq l$. The fact implies that the conditions (1) and (2) are equivalent. The same argument does work well to show the equivalence of (4) and (5). We have the result.

Proposition 3.3. Let $X$ be a simply-connected space as in Theorem 1.3. Then

$$
\operatorname{Gr} H H^{*}\left(C^{*}(X ; \mathbb{Z} / 2) ; C^{*}(X ; \mathbb{Z} / 2)\right) \cong \wedge\left(y_{1}, y_{2}, \ldots, y_{l}\right) \otimes \mathbb{Z} / 2\left[\nu_{1}^{*}, \nu_{2}^{*}, \ldots, \nu_{l}^{*}\right]
$$

as a bigraded algebra, where bideg $y_{j}=\left(0, \operatorname{deg} y_{j}\right)$ and bideg $\nu_{j}^{*}=\left(1,-\operatorname{deg} y_{j}\right)$.

Proof. Since $S q^{1} \equiv 0$ on $H^{*}(X ; \mathbb{Z} / 2)$ by assumption, it follows from [44, Theorem] that the Eilenberg-Moore spectral sequence converging to $H^{*}(L X ; \mathbb{Z} / 2)$ collapses at the $E_{2}$-term. Thanks to Proposition 3.2 , we see that the Moore spectral sequence for $C^{*}(X)$ collapses at the $E_{2}$-term. Proposition 2.4 yields that $E_{\infty}^{*, *}$ is isomorphic to $H^{*}(X) \otimes \mathbb{Z} / 2\left[\nu_{1}^{*}, \ldots, \nu_{l}^{*}\right]$ as a bigraded algebra. This completes the proof.

In the case where the characteristic of the underlying field is odd, we can solve the extension problem in the associated bigraded algebra for an appropriate space.

Proposition 3.4. Let $p$ be odd prime and $G$ a simply-connected $H$-space whose mod $p$ cohomology is an exterior algebra, say $H^{*}(G ; \mathbb{Z} / p) \cong \Lambda\left(y_{1}, \ldots, y_{l}\right)$. Then

$$
H H^{*}\left(C^{*}(G ; \mathbb{Z} / p) ; C^{*}(G ; \mathbb{Z} / p)\right) \cong \wedge\left(y_{1}, y_{2}, \ldots, y_{l}\right) \otimes \mathbb{Z} / p\left[\nu_{1}^{*}, \nu_{2}^{*}, \ldots, \nu_{l}^{*}\right]
$$

as an algebra, where bideg $y_{j}=\left(0, \operatorname{deg} y_{j}\right)$ and bideg $\nu_{j}^{*}=\left(1,-\operatorname{deg} y_{j}\right)$.

Proof. The spectral sequence $\left\{\widetilde{E}_{r}^{*, *}, \widetilde{d}_{r}\right\}$ converging to $H^{*}(L G ; \mathbb{Z} / p)$ collapses at the $E_{2}$-term. In fact, since $G$ is an H-space, it follows that $L G$ is homotopy equivalent to the product $G \times \Omega G$, where $\Omega G$ denotes the based loop space. This implies that

$$
\begin{aligned}
H^{*}(L G ; \mathbb{Z} / p) & \cong H^{*}(G ; \mathbb{Z} / p) \otimes H^{*}(\Omega G ; \mathbb{Z} / p) \\
& \cong \wedge\left(y_{1}, y_{2}, \ldots, y_{l}\right) \otimes \Gamma\left[\nu_{1}, \ldots, \nu_{l}\right] \cong \operatorname{Total} \widetilde{E}_{2}^{* *},
\end{aligned}
$$


where $\operatorname{deg} \nu_{i}=\operatorname{deg} y_{i}-1$. The third isomorphism follows from the usual computation of the $E_{2}$-term with the Koszul-Tate resolution described in Proposition 2.1 By virtue of Proposition 3.2 we see that the Moore spectral sequence also collapses at the $E_{2}$-term. Thus Proposition 2.4 yields that

$$
\operatorname{Gr} H H^{*}\left(C^{*}(G ; \mathbb{Z} / p) ; C^{*}(G ; \mathbb{Z} / p)\right) \cong \wedge\left(y_{1}, y_{2}, \ldots, y_{l}\right) \otimes \mathbb{Z} / p\left[\nu_{1}^{*}, \nu_{2}^{*}, \ldots, \nu_{l}^{*}\right]
$$

as a bigraded algebra. It is immediate that $y_{i}^{2}=0$ in $H H^{*}\left(C^{*}(G ; \mathbb{Z} / p) ; C^{*}(G ; \mathbb{Z} / p)\right)$ for any $i$ because $p$ is odd. We have the result.

\section{The associated Bigraded Batalin-Vilkovisky algebra}

In this section, a Batalin-Vilkovisky algebra structure on the Moore spectral sequence are considered. We first recall the Batalin-Vilkovisky algebra structure on the Hochschild cohomology defined in [34.

Let $M$ be a simply-connected Poincaré duality space of formal dimension $d$ and let $A$ stand for the singular cochain algebra $C^{*}(M ; \mathbb{K})$. Let $\mathbb{B}$ denote the normalized bar complex $\mathbb{B}(A ; A ; A)$. We define an isomorphism of complexes

$$
\iota: \operatorname{Hom}\left(A \otimes_{A \otimes A^{o p}} \mathbb{B}, \mathbb{K}\right) \stackrel{\cong}{\rightarrow} \operatorname{Hom}_{A \otimes A^{o p}}\left(\mathbb{B}, A^{\vee}\right)
$$

by $\iota(f)(\alpha)(a)=(-1)^{|a||\alpha|} f(a \otimes \alpha)$ for $\alpha \in \mathbb{B}$ and $a \in A$. Here the $A$-bimodule structure of $A^{\vee}$ is defined by $\langle f \cdot \alpha \cdot g ; h\rangle=(-1)^{|f|}\langle\alpha ; g h f\rangle$ for $f, g, h \in A$ and $\alpha \in A^{\vee}$. Then one obtains an isomorphism

$$
\left.\iota^{*}: \operatorname{Hom}\left(H\left(A \otimes_{A \otimes A^{o p}} \mathbb{B}\right), \mathbb{K}\right)\right) \underset{\kappa}{\stackrel{\kappa}{\cong}} H\left(\operatorname{Hom}\left(A \otimes_{A \otimes A^{o p}} \mathbb{B}, \mathbb{K}\right)\right) \stackrel{H(\iota)}{\cong} H H^{*}\left(A ; A^{\vee}\right),
$$

where $\kappa$ denotes the Künneth isomorphism. Observe that the source of the map $\iota^{*}$ is the dual $H H_{*}(A ; A)^{\vee}$ to the Hochschild homology $H H_{*}(A ; A)$ of $A$. We also recall the quasi-isomorphism $J: A \otimes_{A \otimes A^{o p}} \mathbb{B} \rightarrow C^{*}(L M)$ of differential graded modules due to Jones 23]. Then it follows that this quasi-isomorphism fits in the commutative diagram

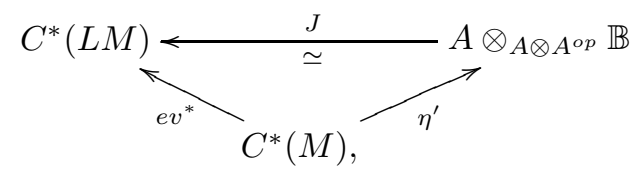

where $e v: L X \rightarrow X$ is the evaluation map at zero and $\eta^{\prime}$ is the chain map defined by $\eta^{\prime}(a)=a \otimes 1$. Therefore we have a commutative diagram

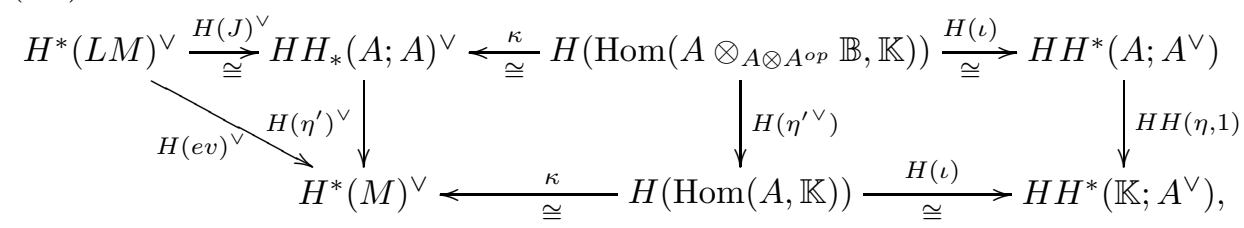

where $\eta: \mathbb{K} \rightarrow A$ denotes the unit. It is readily seen that a section $s: M \rightarrow L M$ of the evaluation map $e v$ induces a section $H(s)^{\vee}$ of the map $H(e v)^{\vee}$. Let $B$ be the Connes boundary map on $A \otimes T(s \bar{A}) \cong A \otimes_{A \otimes A^{o p}} \mathbb{B}$; see [19]. By definition, we see that

$$
B\left(a_{0}\left[a_{1}\left|a_{2}\right| \ldots \mid a_{k}\right]\right)=\sum_{i=0}^{k}(-1)^{\left(\varepsilon_{i}+1\right)\left(\varepsilon_{k+1}-\varepsilon_{i}\right)} 1\left[a_{i}|\ldots| a_{k}\left|a_{0}\right| \ldots \mid a_{i-1}\right] .
$$


We then have

Proposition 4.1. 34, Propositions 11 and 12](i) Let $\omega_{A}^{\vee} \in H(A)^{\vee}$ be the dual base of the fundamental class of $M$. Define an element $[m] \in H H^{-d}\left(A, A^{\vee}\right)$ by $[m]=\iota^{*} H(J)^{\vee} H(s)^{\vee}\left(\omega_{A}^{\vee}\right)$. Then the product $-\smile[m]$ induces an isomorphism

$$
\theta: H H^{p}(A ; A) \rightarrow H H^{p-d}\left(A ; A^{\vee}\right) .
$$

(ii) The Hochschild cohomology ring $H H^{*}(A ; A)$ is a Batalin-Vilkovisky algebra equipped with the $B$ - $V$ operator $\Delta$ of degree -1 defined by the composite

$$
\begin{aligned}
& H H^{p}(A ; A) \stackrel{\theta}{\cong} H H^{p-d}\left(A ; A^{\vee}\right) \stackrel{\iota^{*-1}}{\longrightarrow} H H_{-p+d}(A ; A)^{\vee} \\
& H(B)^{\vee} \downarrow \\
& H H^{p-1}(A ; A) \stackrel{\cong}{\stackrel{\theta}{\longrightarrow}} H H^{p-d-1}\left(A ; A^{\vee}\right) \underset{\iota^{*}}{\longleftarrow} H H_{-p+d+1}(A ; A)^{\vee} .
\end{aligned}
$$

Remark 4.2. Let $H$ be a Poincaré duality algebra with the fundamental class $\omega_{H}$ and $P D: H \stackrel{\cong}{\rightrightarrows} H^{\vee}$ the isomorphism of $H$-bimodules defined by the Poincaré duality; that is, $P D(1)=\omega_{H}^{\vee}$, where $\omega_{H}^{\vee}$ denotes the dual element to $\omega_{H}$. As mentioned in the proof of Proposition 3.2, the map $P D$ induces the isomorphism $H H^{*}(1 ; P D): H H^{*}(H ; H) \stackrel{\cong}{\rightrightarrows} H H^{*}\left(H ; H^{\vee}\right)$. Moreover, it is readily seen that the isomorphism $H H(1 ; P D)$ coincides with the cup product

$$
\smile \omega_{H}^{\vee}: H H^{*}(H ; H) \rightarrow H H^{*}\left(H, H^{\vee}\right)
$$

Here $\omega_{H}^{\vee}$ is considered an element in $H^{\vee} \cong H H^{0, *}\left(H ; H^{\vee}\right)$.

We retain the same notations as in the proof of Theorem 3.1. Let $\widehat{\mathbf{C}}$ and $\overline{\mathbf{C}}$ stand for the Hochschild cochains $\operatorname{Hom}_{A \otimes A^{o p}}\left(\mathbb{B}, A^{\vee}\right)$ and $\operatorname{Hom}_{\mathbb{K} \otimes \mathbb{K}}\left(\mathbb{K}, A^{\vee}\right)$, respectively. We define the same filtrations $\left\{F^{p} \widehat{\mathbf{C}}\right\}$ and $\left\{F^{p} \overline{\mathbf{C}}\right\}$ as that of $\mathbf{C}$. Their filtrations construct the spectral sequences $\left\{\widehat{E}_{r}^{*, *}, \widehat{d}_{r}\right\}$ and $\left\{\bar{E}_{r}^{*, *}, \bar{d}_{r}\right\}$ converging to $H H^{*}\left(A ; A^{\vee}\right)$ and $H H^{*}\left(\mathbb{K}, A^{\vee}\right)=H\left(A^{\vee}\right)$, respectively. We see that $F^{p} \overline{\mathbf{C}}=0$ for $p>0$ and hence $\bar{E}_{r}^{p, *}=0$ for $p>0$. Since the cup product respects the filtrations $\widehat{\mathbf{C}}$ and $\overline{\mathbf{C}}$, it follows that the spectral sequences converge to the targets as algebras. Indeed, the target $A^{\vee}$ of the Hochschild cochains should be replace with $\left(C^{*}(M) / I\right)^{\vee}$ as in the proof of Theorem 3.1 when considering the convergence of the spectral sequence. However to simplify, we also write $A^{\vee}$ for the reduction.

We give a $\mathrm{B}-\mathrm{V}$ algebra structure to the Moore spectral sequence.

Theorem 4.3. Let $M$ be a simply-connected Poincaré duality space. Then the Moore spectral sequence $\left\{E_{r}^{*, *}, d_{r}\right\}$ converging to $H H\left(C^{*}(M) ; C^{*}(M)\right)$ admits the structure of a differential Batalin-Vilkovisky bigraded algebra, in the sense that each term $E_{r}^{*, *}$ is endowed with the $B$-V operator $\Delta_{r}: E_{r}^{p, q} \rightarrow E_{r}^{p-1, q}$ such that $d_{r} \Delta_{r}+$ $\Delta_{r} d_{r}=0, H\left(\Delta_{r}\right)=\Delta_{r+1}$ and $E_{\infty}^{*, *}$ is isomorphic to $\operatorname{Gr} H H^{*}\left(C^{*}(M) ; C^{*}(M)\right)$ as bigraded Batalin-Vilkovisky algebras.

Proof. We first recall that the $E_{r}$-term of the Moore spectral sequence $\left\{E_{r}, d_{r}\right\}$ is defined by $E_{r}^{s, t}=Z_{r}^{s, t} /\left(Z_{r-1}^{s+1, t-1}+B_{r-1}^{s, t}\right)$, where $Z_{r}=F^{s} \mathbf{C}^{s+t} \cap \partial^{-1}\left(F^{s+r} \mathbf{C}^{s+r+1}\right)$ and $B_{r}^{s, t}=F^{s} \mathbf{C}^{s+t} \cap \partial\left(F^{s-r} \mathbf{C}^{s+r-1}\right)$. The $E_{r}$-term $\widehat{E}_{r}^{s, t}$ of the spectral sequence $\left\{\widehat{E}_{r}^{*, *}, \widehat{d}_{r}\right\}$ is defined by the same form with the filtration $\left\{F^{p} \widehat{\mathbf{C}}\right\}$. 
Let $m \in F^{0} \widehat{\mathbf{C}}^{-d}$ be a cocycle representing the element $[m] \in H H^{-d}\left(A, A^{\vee}\right)$ described in Proposition 4.1. Then it follows from [28, Lemma 2.1] that $\{m\}$ is a permanent cycle. The cup product

$$
\smile: \operatorname{Hom}_{A \otimes A^{o p}}\left(\mathbb{B}_{*}, A\right) \otimes \operatorname{Hom}_{A \otimes A^{o p}}\left(\mathbb{B}_{*}, A^{\vee}\right) \rightarrow \operatorname{Hom}_{A \otimes A^{o p}}\left(\mathbb{B}_{*}, A^{\vee}\right)
$$

respects the filtrations; that is, $F^{s} \mathbf{C}^{n} \smile F^{t} \widehat{\mathbf{C}}^{m} \subset F^{s+t} \widehat{\mathbf{C}}^{n+m}$. Therefore the product with the element $\{m\} \in \widehat{E}_{2}^{0,-d} \cong \widehat{E}_{r}^{0,-d}$ induces a morphism

$$
E(m)_{r}:=-\smile\{m\}: E_{r}^{p, q} \rightarrow \widehat{E}_{r}^{p, q-d}
$$

of spectral sequences. We show that $E(m)_{2}$ is an isomorphism and hence so is $E(m)_{r}$ for $2 \leq r \leq \infty$.

We observe that the unit $\eta: \mathbb{K} \rightarrow A$ induces the morphism $\left\{E(\eta)_{r}\right\}:\left\{\widehat{E}_{r}^{*, *}, \widehat{d}_{r}\right\} \rightarrow$ $\left\{\bar{E}_{r}^{*, *}, \bar{d}_{r}\right\}$ of spectral sequences. Consider the map

$$
E(\eta)_{2}: \widehat{E}_{2}^{0,-d} \cong H H^{0,-d}\left(H(A), H\left(A^{\vee}\right)\right) \rightarrow \bar{E}_{2}^{0,-d} \cong H^{-d}\left(A^{\vee}\right) .
$$

Then it follows that the Künneth map $\kappa: H(\operatorname{Hom}(A ; \mathbb{K})) \stackrel{\cong}{\rightrightarrows} \operatorname{Hom}(H(A) ; \mathbb{K})=$ $H(A)^{\vee}$ sends the image $E(\eta)_{2}(\{m\})$ of $\{m\} \in \widehat{E}_{2}^{0,-d}$ to the dual to the fundamental class. To see this, we consider the commutative diagram

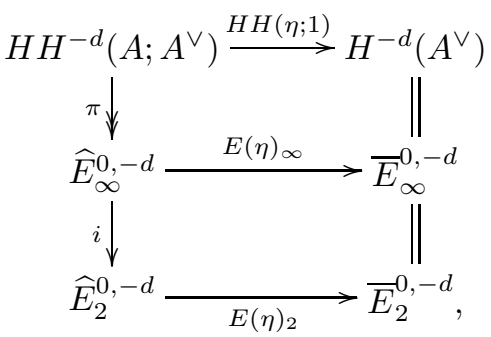

where $\pi$ and $i$ denote the natural projection and the natural inclusion, respectively. Then the definition of $[m]$ and the diagram (4.1) enable us to deduce that

$$
\kappa E(\eta)_{2}(\{m\})=\kappa E(\eta)_{2} i \pi([m])=\kappa H H(\eta ; 1)[m]=\omega_{M}^{\vee} .
$$

Moreover, we see that the map $E(\eta)_{2}: H^{*}\left(A^{\vee}\right) \cong \widehat{E}_{2}^{0, *} \rightarrow \bar{E}_{2}^{0, *}=H^{*}\left(A^{\vee}\right)$ is the identity since $E(\eta)_{2}=H H(H(\eta), 1)$. This implies that $\kappa(\{m\})=\kappa E(\eta)_{2}(\{m\})=$ $\omega_{M}^{\vee}$. Therefore, the map $E(m)_{2}$ coincides with the cup product with $\omega_{M}^{\vee}$. Remark 4.2 allows one to conclude that $E(m)_{2}$ is an isomorphism.

Define a map $\Delta^{\prime}: \widehat{\mathbf{C}} \rightarrow \widehat{\mathbf{C}}$ with degree -1 by $\Delta^{\prime}=\iota \circ B^{\vee} \circ \iota^{-1}$, where $B^{\vee}$ is defined by $B^{\vee}(f)=(-1)^{|f|} f \circ B$. It is readily seen that $\Delta^{\prime}$ maps $F^{p} \widehat{\mathbf{C}}^{n}$ to $F^{p-1} \widehat{\mathbf{C}}^{n-1}$ and hence the map $\Delta^{\prime}$ induces a morphism $\left\{E\left(\Delta^{\prime}\right)_{r}\right\}:\left\{\widehat{E}_{r}^{*, *}, \widehat{d}_{r}\right\} \rightarrow\left\{\widehat{E}_{r}^{*, *}, \widehat{d}_{r}\right\}$ of spectral sequences with bidegree $(-1,0)$. Thus we define maps $\Delta_{r}: E_{r}^{*, *} \rightarrow E_{r}^{*, *}$ by $\Delta_{r}=E(m)_{r}^{-1} \circ \Delta_{r}^{\prime} \circ E(m)_{r}$ for $r \geq 2$, which give the morphism of spectral sequences from $\left\{E_{r}^{*, *}, d_{r}\right\}$ to itself with bidegree $(-1,0)$. Proposition 4.1(ii) implies that the $E_{2}$-term of the Moore spectral sequence admits the structure of a Batalin-Vilkovisky algebra with the operator $\Delta_{2}$. The equality $b B+B b=0$ for the differential $b$ of the Hochschild complex $A \otimes T(s \bar{A})$ enables us to deduce that $d_{r} \Delta_{r}+\Delta_{r} d_{r}=0$ and that $H\left(\Delta_{r}\right)=\Delta_{r+1}$.

The isomorphism $E_{\infty}^{*, *} \stackrel{\cong}{\rightarrow} \operatorname{Gr} H H^{*}\left(C^{*}(M) ; C^{*}(M)\right)$ is induced by the inclusion of cocycles. Therefore the isomorphism is compatible with the $\mathrm{B}-\mathrm{V}$ operators. Observe that the filtration $\left\{F^{p} H H^{n}\right\}$ of $H H^{n}(A ; A)$ and the filtration $\left\{F^{p} \widehat{H}^{n}\right\}$ 
of $H H^{n}\left(A ; A^{\vee}\right)$ are bounded for each $n$ since $H^{*}(M)$ is of finite dimension; see the proof of Theorem 3.1. Recall that $E(m)_{\infty}: E_{\infty}^{*, *} \rightarrow \widehat{E}_{\infty}^{* * *}$ is an isomorphism. This yields that the map from $\left\{F^{p} H H^{n}\right\}$ to $\left\{F^{p} \widehat{H}^{n}\right\}$ induced by $\theta$ is also an isomorphism. This completes the proof.

We have fundamental properties of the Moore spectal sequence.

Corollary 4.4. With the same notations as in the proof of Theorem 4.3, one obtains that

(i) the map

$$
E(\eta)_{2}: \widehat{E}_{2}^{0, *} \cong H H^{0, *}\left(H(A), H\left(A^{\vee}\right)\right)=H^{*}\left(A^{\vee}\right) \rightarrow \bar{E}_{2}^{0, *} \cong H^{*}\left(A^{\vee}\right)
$$

is the identity and the element $\{m\} \in \widehat{E}_{2}^{0,-d}$ is the dual to the fundamental class $\omega_{M}$ in $H(A)=H^{*}(M ; \mathbb{K})$, and that

(ii) each element in $E_{2}^{0, *}$ is a permanent cycle.

Proof. The assertion (i) follows from the argument with the diagram (4.2) in the proof of Theorem 4.3 .

We prove the assertion (ii). It suffices to show that all the elements in $\widehat{E}_{2}^{0, *}$ are permanent cycles because $E(m)_{r}$ is an isomorphism for any $r \geq 2$. The result follows from the commutative diagram

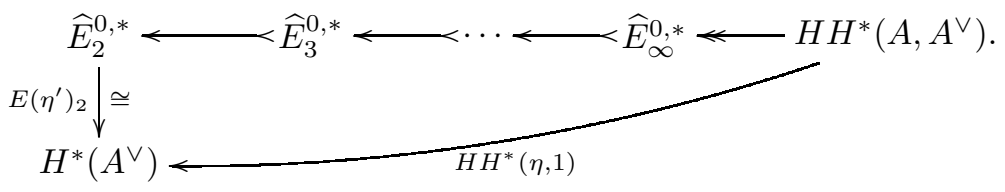

In fact, since the evaluation map $L M \rightarrow M$ admits a section, it follows from the diagram (4.1) that the map $H H^{*}(\eta, 1)$ is an epimorphism. We have the result.

In order to prove Theorem [1.3, we look at the behavior of the B-V operator on the $E_{2}$-term of the Moore spectral sequence. To this end, we give an explicit isomorphism between the Hochschild homology of a graded module computed by the bar resolution and that computed by the Koszul-Tate resolution described in Proposition 2.1.

Lemma 4.5. Let $A$ be an exterior algebra over a field of characteristic 2, say $A \cong \wedge\left(y_{1}, \ldots, y_{l}\right)$. Then there exists an isomorphism

$$
\phi: H(A \otimes T(s \bar{A}), b) \rightarrow H\left(A \otimes_{A \otimes A} \mathcal{F}, 1 \otimes d\right) \cong \wedge\left(y_{1}, \ldots, y_{l}\right) \otimes \Gamma\left[\nu_{1}, \ldots, \nu_{l}\right]
$$

such that $\phi\left(\left[y_{i}\right]\right)=\nu_{i}, \phi\left(\left[y_{i} \mid y_{i}\right]\right)=\gamma_{2}\left(\nu_{i}\right)$ and $\phi\left(\left[y_{i} \mid y_{j}\right]+\left[y_{j} \mid y_{i}\right]\right)=\nu_{i} \nu_{j}$ for $i \neq j$. Here $(A \otimes T(s \bar{A}), b)$ and $(\mathcal{F}, d)$ denote the Hochschild complex and the resolution of $A$ mentioned in Proposition 2.1, respectively.

Proof. We construct a DG $A \otimes A^{o p}$-module map $\xi=\left\{\xi_{i}\right\}: \mathbb{B}(A ; A ; A) \rightarrow \mathcal{F}$ which covers the identity map on $A$ by induction on the filtration degree.

Let $\mathcal{F}_{i}$ denote a submodule of the form $\{x \in \mathcal{F} \mid$ bideg $x=(-i, *)\}$. Suppose that a map $u: Z_{i} \rightarrow \mathcal{F}_{i}$ satisfies the condition that $\partial u=\xi_{i-1} \partial$, where $Z_{i}$ is an $A \otimes A^{o p}$-module which is a direct summand of $\mathbb{B}_{i}$; that is, $\mathbb{B}_{i}=Z_{i} \oplus Z_{i}^{\prime}$ for some $A \otimes A^{o p}$-submodule $Z_{i}^{\prime}$. Since $\mathbb{B}_{i}$ is a free $A \otimes A^{o p}$-module and $\mathcal{F}$ is acyclic, it follows that there exists an $A \otimes A^{o p}$-module map $\xi_{i}: \mathbb{B}_{i} \rightarrow \mathcal{F}_{i}$ such that $\left.\xi_{i}\right|_{Z_{i}}=u$ and $\partial \xi_{i}=\xi_{i-1} \partial$. Observe that $Z_{i}^{\prime}$ is projective. 
Define $\xi_{0}: A \otimes A=\mathbb{B}_{0}(A ; A ; A) \rightarrow \mathcal{F}_{0}=A \otimes A^{o p}$ to be the identity map and $\xi_{1}: \mathbb{B}_{1}(A ; A ; A)=A \otimes T^{1}(s \bar{A}) \otimes A \rightarrow A \otimes A \otimes \mathbb{K}\left\{\nu_{i} ; i=1, . ., l\right\}$ by extending the $A \otimes A$-module map which sends basis $\left[y_{i}\right]$ to $\nu_{i}$. We observe that $d \xi_{1}\left(\left[y_{i}\right]\right)=d \nu_{i}=$ $y_{i} \otimes 1+1 \otimes y_{i}=\xi_{0} d\left(\left[y_{i}\right]\right)$. A direct computation shows that

$$
\begin{aligned}
\xi_{1} d\left(\left[y_{i} \mid y_{j}\right]+\left[y_{j} \mid y_{i}\right]\right) & =\left(y_{i} \otimes 1+1 \otimes y_{i}\right) \nu_{j}+\left(y_{j} \otimes 1+1 \otimes y_{j}\right) \nu_{i} \text { for } i \neq j \text { and } \\
\xi_{1} d\left(\left[y_{i} \mid y_{i}\right]\right) & =\left(y_{i} \otimes 1+1 \otimes y_{i}\right) \nu_{i} .
\end{aligned}
$$

This enables us to define an $A \otimes A^{o p}$-module map $\xi_{2}: \mathbb{B}_{2}(A ; A ; A) \rightarrow \mathcal{F}_{2}$ which is compatible with the differentials and satisfy the condition that $\xi_{2}\left(\left[y_{i} \mid y_{i}\right]\right)=\gamma_{2}\left(\nu_{i}\right)$ and $\xi_{2}\left(\left[y_{i} \mid y_{j}\right]+\left[y_{j} \mid y_{i}\right]\right)=\nu_{i} \nu_{j}$. We define $\xi: \mathbb{B}(A ; A ; A) \rightarrow \mathcal{F}$ extending $\left\{\xi_{i}\right\}_{0 \leq i \leq 2}$ to the whole complex $\mathbb{B}(A ; A ; A)$.

Let $\phi^{\prime}: A \otimes T(s \bar{A}) \rightarrow A_{A \otimes A^{o p}} \mathbb{B}(A ; A ; A)$ be an isomorphism of complexes defined by $\phi^{\prime}\left(a_{0}\left[a_{1}|\ldots| a_{n}\right]\right)=a_{0} \otimes 1\left[a_{1}|\ldots| a_{n}\right] 1$. The induced map $\phi=H\left((1 \otimes \xi) \circ \phi^{\prime}\right)$ : $H(A \otimes T(s \bar{A}), b) \rightarrow H\left(A \otimes_{A \otimes A} \mathcal{F}, 1 \otimes d\right)$ is the desired isomorphism.

Proof of Theorem 1.3. It follows from the proof of Proposition 3.3 that as bigraded algebras

$$
\operatorname{Gr} H H^{*}\left(C^{*}(X ; \mathbb{Z} / 2) ; C^{*}(X ; \mathbb{Z} / 2)\right) \cong E_{2}^{*, *} \cong \wedge\left(y_{1}, y_{2}, \ldots, y_{l}\right) \otimes \mathbb{Z} / 2\left[\nu_{1}^{*}, \nu_{2}^{*}, \ldots, \nu_{l}^{*}\right],
$$

where bideg $y_{j}=\left(0, \operatorname{deg} y_{j}\right)$ and bideg $\nu_{j}^{*}=\left(1,-\operatorname{deg} y_{j}\right)$. Thus in order to prove Theorem 1.3, it suffices to determine the B-V structure on the $E_{2}$-term of the Moore spectral sequence.

Let $H$ denote the cohomology $H^{*}(M)$ and let $\omega_{H}$ be the fundamental class, namely $\omega_{H}=y_{1} \cdots y_{l}$. We first recall the isomorphism of complexes

$$
\iota: \operatorname{Hom}\left(H \otimes_{H \otimes H^{o p}} \mathcal{F}, \mathbb{K}\right) \rightarrow \operatorname{Hom}_{H \otimes H^{o p}}\left(\mathcal{F}, H^{\vee}\right)
$$

defined by $\iota(f)(\alpha)(a)=(-1)^{|a||\alpha|} f(a \otimes \alpha)$ for $a \in H$ and $\alpha \in \mathcal{F}$, where $\mathcal{F} \rightarrow$ $H \rightarrow 0$ is the Koszul-Tate resolution of $H$ mentioned in Proposition 2.1. Recall also the $H$-bimodule structure on $H^{\vee}$; see the beginning of this section. Using the isomorphism $\iota$, the cup product $-\smile \omega_{H}^{\vee}$ and $\phi$ in Lemma 4.5 we can determine the $\mathrm{B}-\mathrm{V}$ operator on the $E_{2}$-term. The key to the computation is that the Connes boundary map $B$ is a derivation on cycles modulo boundary with respect to the shuffle product on the Hochschild complex. By virtue of Corollary 4.4 (i), we have $\theta\left(\nu_{i}^{*} \nu_{j}^{*}\right)=\nu_{i}^{*} \nu_{j}^{*} \smile m=\nu_{i}^{*} \nu_{j}^{*} \smile \omega_{H}^{\vee}=\omega_{H}^{\vee} \nu_{i}^{*} \nu_{j}^{*}$, which sends $\nu_{i} \nu_{j}$ to $\omega_{H}^{\vee} \in H^{\vee}$. Moreover, by the definition of $\iota$, one obtains

$$
\iota\left(\left(\omega_{H} \nu_{i} \nu_{j}\right)^{*}\right)\left(\nu_{i} \nu_{j}\right)(a)=\left(\omega_{H} \nu_{i} \nu_{j}\right)^{*}\left(a \nu_{i} \nu_{j}\right)= \begin{cases}1 & \text { if } a=\omega_{H} \\ 0 & \text { otherwise. }\end{cases}
$$

This yields that $\theta\left(\nu_{i}^{*} \nu_{j}^{*}\right)=\iota\left(\omega_{H} \nu_{i} \nu_{j}\right)^{*}$. We then have

$$
\begin{aligned}
& \left\langle H(B)^{\vee} \phi^{\vee}\left(\omega_{H} \nu_{i} \nu_{j}\right)^{*}, y_{i_{1}} \cdots y_{i_{u}}\left[y_{t}\right]\right\rangle \\
= & \left\langle\left(\omega_{H} \nu_{i} \nu_{j}\right)^{*}, \phi H(B)\left(y_{i_{1}} \cdots y_{i_{u}}\left[y_{t}\right]\right)\right\rangle \\
= & \left\langle\left(\omega_{H} \nu_{i} \nu_{j}\right)^{*}, \phi \sum_{i_{s}} y_{i_{1}} \cdots \check{y}_{i_{s}} \cdots y_{i_{u}}\left[y_{i_{s}}\right] *\left[y_{t}\right]\right\rangle \\
= & \left\langle\left(\omega_{H} \nu_{i} \nu_{j}\right)^{*}, \sum_{i_{s} \neq t} y_{i_{1}} \cdots \check{y}_{i_{s}} \cdots y_{i_{u}} \phi\left(\left[y_{i_{s}} \mid y_{t}\right]+\left[y_{t} \mid y_{i_{s}}\right]\right)\right\rangle \\
= & \left\langle\left(\omega_{H} \nu_{i} \nu_{j}\right)^{*}, \sum_{i_{s} \neq t} y_{i_{1}} \cdots \check{y}_{i_{s}} \cdots y_{i_{u}} \nu_{i_{s}} \nu_{t}\right\rangle=0 .
\end{aligned}
$$


Here $*$ denotes the shuffle product on the Hochschild homology and $\check{y}_{j}$ means that the element $y_{j}$ has been deleted. The second equality follows from the fact that $H(B)$ is a derivation with respect to the shuffle product; see [19, Lemma 4.3]. In fact, we see that

$$
\begin{aligned}
\left.H(B)\left(y_{i_{1}} \cdots y_{i_{u}}\left[y_{t}\right]\right)\right)= & H(B)\left(y_{i_{1}} * \cdots * y_{i_{u}} *\left[y_{t}\right]\right) \\
= & \left(H(B) y_{i_{1}}\right) * y_{i_{2}} * \cdots * y_{i_{u}} *\left[y_{t}\right] \\
& +\cdots+y_{i_{1}} * \cdots * y_{i_{u-1}} *\left(H(B) y_{i_{u}}\right) *\left[y_{t}\right] \\
& +y_{i_{1}} * \cdots * y_{i_{u}} *\left(H(B)\left[y_{t}\right]\right) \\
= & \sum_{i_{s}} y_{i_{1}} * \cdots *\left[y_{i_{s}}\right] * \cdots * y_{i_{u}} *\left[y_{t}\right]+y_{i_{1}} * \cdots * y_{i_{u}} * 0 \\
= & \sum_{i_{s}} y_{i_{1}} \cdots \check{y}_{i_{s}} \cdots y_{i_{u}}\left[y_{i_{s}}\right] *\left[y_{t}\right] .
\end{aligned}
$$

Thus we have $\Delta\left(\nu_{i}^{*} \nu_{j}^{*}\right)=0$.

We compute $\Delta\left(y_{j} \nu_{i}^{*}\right), \Delta\left(\nu_{i}^{*}\right)$ and $\Delta\left(y_{i}\right)$ below. Since $\omega_{H}^{\vee} \cdot y_{j}=\left(y_{1} \cdots \check{y}_{j} \cdots y_{l}\right)^{*}$ in $H^{\vee}$, it follows that

$$
\begin{aligned}
\theta\left(y_{j} \nu_{i}^{*}\right) & =\omega_{H}^{\vee}\left(y_{j} \nu_{i}^{*}\right) \\
& =\left(y_{1} \cdots \check{y}_{j} \cdots y_{l}\right)^{*} \nu_{i}^{*} \\
& =\iota\left(\left(y_{1} \cdots \check{y}_{j} \cdots y_{l} \nu_{i}\right)^{*}\right) .
\end{aligned}
$$

Moreover, we see that

$$
\begin{aligned}
& \left\langle H(B)^{\vee} \phi^{\vee}\left(\left(y_{1} \cdots \check{y}_{j} \cdots y_{l} \nu_{i}\right)^{*}\right), \omega_{H}\right\rangle \\
= & \left\langle\left(y_{1} \cdots \check{y}_{j} \cdots y_{l} \nu_{i}\right)^{*}, \phi \sum_{t=1}^{l}\left(y_{1} \cdots \check{y}_{t} \cdots y_{l}\right)\left[y_{t}\right]\right\rangle=\delta_{i j} .
\end{aligned}
$$

The fact that $\theta(1)=\omega_{H}^{\vee}$ allows us to deduce that $\Delta\left(y_{j} \nu_{i}^{*}\right)=\delta_{i j} \cdot 1$. For dimensional reasons, we have $\Delta\left(\nu_{i}^{*}\right)=\Delta\left(y_{j}\right)=\Delta\left(y_{i} y_{j}\right)=0$. This completes the proof.

The following corollary illustrates that the Moore spectral sequence is reliable when calculating explicitly the Hochschild cohomology of the singular cochain on a space.

Corollary 4.6. Let $M$ be a simply-connected mod 2 Poincaré duality space whose mod 2 cohomology is isomorphic to an exterior algebra of the form $\wedge\left(y_{1}, y_{2}\right)$, where $\operatorname{deg} y_{1}=\operatorname{deg} y_{2}=n$. Suppose that $n>4$. Then as a Batalin-Vilkovisky algebra

$$
H H^{*}\left(C^{*}(M ; \mathbb{Z} / 2) ; C^{*}(M ; \mathbb{Z} / 2)\right) \cong \wedge\left(y_{1}, y_{2}\right) \otimes \mathbb{Z} / 2\left[\nu_{1}^{*}, \nu_{2}^{*}\right]
$$

in which $\Delta\left(y_{j}\right)=0, \Delta\left(y_{j} y_{j}\right)=0, \Delta\left(\nu_{i}^{*}\right)=0, \Delta\left(\nu_{i}^{*} \nu_{j}^{*}\right)=0$ for $1 \leq i, j \leq l$ and $\Delta\left(y_{i} \nu_{j}^{*}\right)=\delta_{i j} \cdot 1$, where $\operatorname{deg} y_{j}=n$ and $\operatorname{deg} \nu_{j}^{*}=-n+1$ for $j=1$ and 2 .

Proof. By virtue of Theorem 1.3, we see that as bigraded Batalin-Vilkovisky algebras

$$
\operatorname{Gr} H H^{*}\left(C^{*}(M ; \mathbb{Z} / 2) ; C^{*}(M ; \mathbb{Z} / 2)\right) \cong E_{\infty}^{*, *} \cong \wedge\left(y_{1}, y_{2}\right) \otimes \mathbb{Z} / 2\left[\nu_{1}^{*}, \nu_{2}^{*}\right]
$$

with $\Delta\left(y_{j}\right)=0, \Delta\left(\nu_{i}^{*}\right)=0, \Delta\left(\nu_{i}^{*} \nu_{j}^{*}\right)=0$ for $1 \leq i, j \leq l$ and $\Delta\left(y_{i} \nu_{j}^{*}\right)=\delta_{i j} \cdot 1$. Observe that $S q^{1}=0$ on $H^{*}(M ; \mathbb{Z} / 2)=\wedge\left(y_{1}, y_{2}\right)$. 
We have to solve extension problems on the product and on the B-V operator. Since there exists no nonzero element in $E_{\infty}^{p, q}$ for $p \geq 1$ and $p+q=2 n$, it follows that $y_{i}^{2}=0$ for $i=1$ and 2 ; see the figure displayed below.

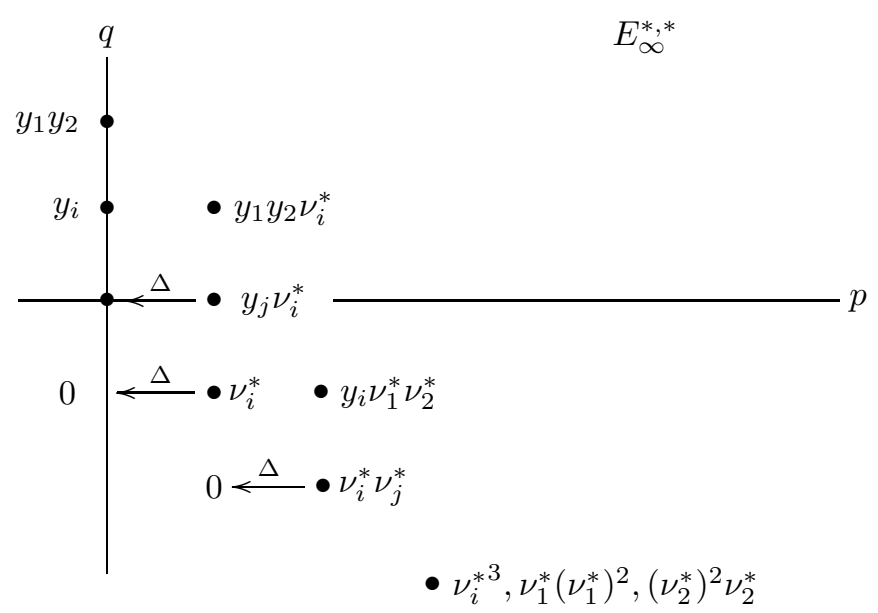

We consider the extension problems on the B-V operator. For non-positive integers $l$ and $m$ with $l+m \geq 3$, we see that

$$
\begin{aligned}
\operatorname{deg} y_{i} \nu_{1}^{* l} \nu_{2}^{* m}<\operatorname{deg} y_{1} y_{2} \nu_{1}^{* l} \nu_{2}^{* m} & =2 n+(l+m)(-n+1) \\
& =2+(l+m-2)(-n+1) \leq 2+(-n+1)<0,
\end{aligned}
$$

$\operatorname{deg} y_{i} \nu_{1}^{*} \nu_{2}^{*}=-n+2, \operatorname{deg} y_{1} y_{2} \nu_{1}^{*} \nu_{2}^{*}=2, \operatorname{deg} y_{i} \nu_{j}^{*}=1$ and $\operatorname{deg} y_{1} y_{2} \nu_{i}^{*}=n+1$. This enables us to conclude that $\Delta\left(y_{i} \nu_{j}^{*}\right)=\delta_{i j} \cdot 1, \Delta\left(y_{j}\right)=0$ and $\Delta\left(y_{i} y_{j}\right)=0$ on $H H^{*}\left(C^{*}(M) ; C^{*}(M)\right)$ because $\operatorname{deg} \Delta\left(y_{i} y_{j}\right)=2 n-1, \operatorname{deg} \Delta\left(y_{i}\right)=n-1$ and $\operatorname{deg} \Delta\left(y_{i} \nu_{j}^{*}\right)=0$. For $l \geq 3$, one has

$$
\begin{aligned}
\operatorname{deg} y_{1} y_{2} \nu_{i}^{* l}-\left(\operatorname{deg} \nu_{1}^{*} \nu_{2}^{*}-1\right) & =2 n+l(-n+1)-2(-n+1)+1 \\
& =\left\{\begin{array}{ll}
n+2 & \text { if } l=3 \\
3 & \text { if } l=4 \\
\text { a negative integer } & \text { if } l>4
\end{array} .\right.
\end{aligned}
$$

Moreover, we see that

$$
\begin{aligned}
\operatorname{deg} y_{i} \nu_{j}^{* l}-\left(\operatorname{deg} \nu_{1}^{*} \nu_{2}^{*}-1\right) & =2 n+l(-n+1)-2(-n+1)+1-n \\
& = \begin{cases}2 & \text { if } l=3 \\
3-n & \text { if } l=4 \\
\text { a negative integer } & \text { if } l>4 .\end{cases}
\end{aligned}
$$

Since $E_{\infty}^{2, q}=0$ for $q<-2 n$ and $\Delta\left(\nu_{1}^{*} \nu_{2}^{*}\right)=0$ in $E_{\infty}^{1,-2 n}$, it follows that $\Delta\left(\nu_{1}^{*} \nu_{2}^{*}\right)=0$ on $H H^{*}\left(C^{*}(M) ; C^{*}(M)\right)$. The same calculation as above shows that

$$
\operatorname{deg} y_{1} y_{2} \nu_{i}^{* l}-\left(\operatorname{deg} \nu_{i}^{*}-1\right)= \begin{cases}n+2 & \text { if } l=2 \\ 3 & \text { if } l=3 \\ \text { a negative integer } & \text { if } l>3 .\end{cases}
$$

and that

$$
\operatorname{deg} y_{i} \nu_{j}^{* l}-\left(\operatorname{deg} \nu_{i}^{*}-1\right)= \begin{cases}2 & \text { if } l=2 \\ 3-n & \text { if } l=3 \\ \text { a negative integer } & \text { if } l>3 .\end{cases}
$$


These deduce that $\Delta\left(\nu_{i}^{*}\right)=0$ on $H H^{*}\left(C^{*}(M) ; C^{*}(M)\right)$. We have the result.

Remark 4.7. In the case $n=3$, we write $\Delta\left(y_{j} \nu_{i}^{*}\right)=\delta_{i j} \cdot 1+\varepsilon y_{1} y_{2} \nu_{1}^{* l} \nu_{2}^{* m}$, where $l+m=3$. The filtration argument we use above does not work well to determine whether $\varepsilon$ is zero or not.

Acknowledgments. The author thanks Hiroshi Nagase for useful comments on the ordinary Hochschild cohomology rings of a polynomial algebra and an exterior algebra. He also thanks the referee for careful reading of a previous version of this paper and for valuable suggestions to revise the article.

\section{APPENDiX}

In this short section, we summarize the notion of "a ring homomorphism" from the Hochschild cohomology to the graded center of a triangulated category. Though there is no result yet, we have a certain expectation that string topology plays a crucial role in the study of global structure of the cochain algebra on a space via the homomorphism.

We begin by recalling the definition of the graded center of a triangulated category.

Definition 5.1. (cf. 4, 3.2], 32, §2]) Let $\mathcal{T}$ be a $\mathbb{K}$-linear triangulated category with suspension functor $\Sigma$. The graded center $\mathcal{Z}(\mathcal{T})$ is a graded family whose degree $n$ component $\mathcal{Z}^{n}(\mathcal{T})$ consists of all natural transformations $\varphi: I d_{\mathcal{T}} \rightarrow \Sigma^{n}$ such that $\varphi \Sigma=(-1)^{n} \Sigma \varphi$.

Let $R$ be a commutative graded ring and $\Phi: R \rightarrow \mathcal{Z}(\mathcal{T})$ a ring homomorphism preserving the degree. Here we ignore set theoretic issues on the graded center. Indeed, the ring homomorphism means that, for each object $X$ in $\mathcal{T}$, one has a homomorphism of graded algebra $\Phi_{X}: R \rightarrow \operatorname{End}_{\mathcal{T}}^{*}(X)$ such that

$$
\Phi_{Y}(\alpha) \beta=(-1)^{|\alpha||\beta|} \beta \Phi_{X}(\alpha)
$$

for $\alpha \in R$ and $\beta \in \operatorname{Hom}_{\mathcal{T}}^{*}(X, Y)$.

Let $A$ be a DG algebra over a field $\mathbb{K}$. Then we have a triangulated category $\mathrm{D}(A)$, which is the derived category of DG modules over $A$ with the shift functor $\Sigma ;(\Sigma N)^{n}=N^{n+1}$, as the suspension functor. It follows from [5, Proposition 1.1] that the cup product on $H H^{*}(A ; A)$ coincides with the Yoneda product. We then have a ring homomorphism $\Phi$ from the Hochschild cohomology ring $H H^{*}(A ; A)$ to the graded center of the triangulated category $\mathrm{D}(A)$. In fact, the homomorphism $\Phi: H H^{*}(A ; A) \rightarrow \mathcal{Z}(\mathrm{D}(A))$ is defined by

$$
\Phi(f)(M)=\Phi_{M}(f)=I d_{M} \otimes_{A} f: M \rightarrow \Sigma^{n} M
$$

in $\mathrm{D}(A)$ for $f \in H H^{n}(A, A)$.

Let $X$ be a simply-connected space whose cohomology with coefficients in $\mathbb{K}$ is locally finite. The general argument above gives a ring homomorphism

$$
\Phi: H H^{*}\left(C_{*}(\Omega X), C_{*}(\Omega X)\right) \rightarrow \mathcal{Z}\left(\mathrm{D}\left(C_{*}(\Omega X)\right)\right) .
$$

By virtue of [11, Theorem I], we have a quasi-isomorphism of DG algebras from the cobar complex of $C_{*}(X)$ to $C_{*}(\Omega X)$. Thus the result [16, Theorem 1] due to Félix, Menichi and Thomas allows one to obtain an isomorphism of algebras between $H H^{*}\left(C_{*}(\Omega X), C_{*}(\Omega X)\right)$ and $H H^{*}\left(C^{*}(X), C^{*}(X)\right)$, which indeed respects the Gerstanhaber algebra structure. Suppose further that $X$ is a closed oriented 
manifold. Then we have a ring homomorphism form the loop homology $\mathbb{H}_{*}(L X)$ to the graded center of the derived category $\mathrm{D}\left(C_{*}(\Omega X)\right)$ with the isomorphism between the loop homology and the Hochschild homology [8, 9, 18, 35].

The same way allows us to define a ring homomorphism

$$
\mathbb{H}_{*}(L X) \cong H H^{*}\left(C^{*}(X), C^{*}(X)\right) \rightarrow \mathcal{Z}\left(\mathrm{D}\left(C^{*}(X)\right)\right) .
$$

Thus it is expected that the loop homology is of great use when studying triangulated categories associated with cochain algebras on spaces via the theory of support varieties; see for example [2, 3, 42, and when considering the (co)chain type level [30, 31] of a space over a simply-connected manifold. Topological properties of spaces, which the graded center captures, remains to be elucidated.

\section{REFERENCES}

[1] L. L. Avramov, R. -O. Buchweitz, S. B. Iyengar and C. Miller, Homology of perfect complexes, Adv. Math. 223(2010), 1731-1781. arXiv: math.AC/0609008v2.

[2] D. Benson, S. B. Iyengar and H. Krause, Local cohomology and support for triangulated categories, Ann. Sci. Éc. Norm. Supér 41(2008), 573-619.

[3] D. Benson, S. B. Iyengar and H. Krause, Stratifying triangulated categories, preprint (2009).

[4] R. -O. Buchweitz and H. Flenner, Global Hochschild (co-)homology of singular spaces, Advances in Math. 217(2008), 205-242.

[5] R. -O. Buchweitz, E. L. Green, N. Snashell and Ø. Solberg, Multiplicative structures for Koszul algebras, preprint (2005), arXiv.org/abs/math/0508177,

[6] M, Chas and D. Sullivan, String topology, preprint (1999), arXiv.org/abs/math/9911159, to appear in Ann. of Math.

[7] C. Cibils and A. Solotar, The Buenos Aires Cyclic Homology Group, Cyclic homology of algebras with one generator, K-theory 5(1991), 51-69.

[8] R. Cohen, Multiplicative properties of Atiyah duality, Homology Homotopy Appl. 6(2004), 269-281.

[9] R. L. Cohen and J. D. S. Jones, A homotopy theoretic realization of string topology, Math. Ann. 324(2002), 773-798.

[10] Y. Félix, S. Halperin and J. -C. Thomas, Gorenstein spaces, Adv. in Math. 71(1988), 92-112.

[11] Y. Félix, S. Halperin and J. -C. Thomas, Adams' cobar equivalence, Trans. Amer. Math. Soc. 329(1992), 531-549.

[12] Y. Félix, S. Halperin and J. -C. Thomas, Differential graded algebras in topology, in: I.M. James (Ed.), Handbook of Algebraic Topology, Elsevier, Amsterdam, 1995, pp. 829-865.

[13] Y. Félix, S. Halperin and J. -C. Thomas, Rational Homotopy Theory, Graduate Texts in Mathematics 205, Springer-Verlag.

[14] Y. Félix and J. -C. Thomas, Rational BV-algebra in string topology, Bull. Soc. Math. France 136(2008), 311-327.

[15] Y. Félix and J. -C. Thomas, String topology on Gorenstein spaces, Math. Ann. 345(2009), 417-452.

[16] Y. Félix, L. Menichi and J. -C. Thomas, Gerstenhaber duality in Hochschild cohomology, J. Pure Appl. Algebra 199(2005), 43-59.

[17] Y. Félix, J. -C. Thomas and M. Vigué-Poirrier, The Hochschild cohomology of a closed manifold. Publ. Math. Inst. Hautes Études Sci. 99(2004), 235-252.

[18] Y. Félix, J. -C. Thomas and M. Vigué-Poirrier, Rational string topology. J. Eur. Math. Soc. (JEMS) 9(2007), 123-156.

[19] E. Getzler and J. D. S. Jones, $A_{\infty}$-algebras and the cyclic bar complex, Illinois J. Math. 34(1990), 256-283.

[20] S. Halperin and J. -M. Lemaire, Notions of category in differential algebra, Algebraic Topology: Rational Homotopy, Springer Lecture Notes in Math., Vol. 1318, Springer, Berlin, New York, 1988, pp. 138-154.

[21] Y. Han and Y. Xu, Hochschild (co)homology of exterior algebras, Comm. Algebra 35(2007), 115-131.

[22] T. Holm, Hochschild cohomology rings of algebras $k[X] /(f)$, Beiträge Algebra Geom. 41(2000), 291-301. 
[23] J. D. S. Jones, Cyclic homology and equivariant homology, Invent. Math. 87(1987), 403-423.

[24] P. Jørgensen, Auslander-Reiten theory over topological spaces, Comment. Math. Helv. 79(2004), 160-182.

[25] P. Jørgensen, The Auslander-Reiten quiver of a Poincaré duality space, Algebr. Represent. Theory 9(2006), 323-336.

[26] R. M. Kaufmann, A proof of a cyclic version of Deligne's conjecture via cacti, Math. Res. Lett. 15 (2008), 901-921.

[27] R. M. Kaufmann, Moduli space actions on the Hochschild co-chains of a Frobenius algebra. II. Correlators, J. Noncommut. Geom. 2(2008), 283-332.

[28] D. Kraines and C. Schochet, Differentials in the Eilenberg-Moore spectral sequence, J. Pure Appl. Algebra 2(1972), 131-148.

[29] K. Kuribayashi, On the mod $p$ cohomology of the spaces of free loops on the Grassmann and Stiefel manifolds, J. Math. Soc. Japan 43(1991), 331-346.

[30] K. Kuribayashi, On the levels of spaces and topological realization of objects in a triangulated category, preprint (2010).

[31] K. Kuribayashi, Upper and lower bounds of the (co)chain type level of a space, preprint (2010), arXiv: math.AT/1006.2669.

[32] M. Linckelmann, On the graded centers and block cohomology, Proc. Edinburgh Math. Soc, 52(2009), 489-514.

[33] L. Menichi, String topology for spheres. With an appendix by Gerald Gaudens and Menichi, Comment. Math. Helv. 84(2009), 135-157.

[34] L. Menichi, Batalin-Vilkovisky algebra structures on Hochschild cohomology, Bull. Soc. Math. France 137(2009), 277-295.

[35] S. A. Merkulov, De Rham model for string topology, Int. Math. Res. Not. 2004, no. 55, $2955-2981$.

[36] M. Mimura and H. Toda, Topology of Lie groups I, Translations of Mathematical Monographs, 91, American Mathematical Society, Providence, RI, 1991.

[37] H. J. Munkholm, The Eilenberg-Moore spectral sequence and strongly homotopy multiplicative maps, J. Pure Appl. Algebra 5(1974), 1-50.

[38] R. Rouquier, Dimensions of triangulated categories, J. K-Theory 1(2008), 193-256.

[39] K. Sanada, On the Hochschild cohomology of crossed products, Comm. Algebra 21(1993), $2727-2748$.

[40] K. Schmidt, Families of Auslander-Reiten components for simply connected differential graded algebras, Math. Z. 426(2010), 43-62.

[41] S. F. Siegel and S. J. Witherspoon, The Hochschild cohomology ring of a group algebra, Proc. London Math. Soc. (3) 79(1999), 131-157.

[42] N. Snashall and $\varnothing$. Solberg, Support varieties and Hochschild cohomology ring, Proc. London Math. Soc. (3) 88(2004), 705-732.

[43] L. Smith, On the characteristic zero cohomology of the free loop space, Amer. J. Math. 103(1981), 887-910.

[44] L. Smith, The Eilenberg-Moore spectral sequence and mod 2 cohomology of certain free loop spaces, Illinois J. Math. 28(1984), 516-522.

[45] T. Thomas and M. Zeinalian, Infinity structure of Poincaré duality spaces, Appendix A by Dennis Sullivan, Algebr. Geom. Topol. 7(2007), 233-260.

[46] T. Tradler, The Batalin-Vilkovisky algebra on Hochschild cohomology induced by infinity inner products, Ann. Inst. Fourier (Grenoble) 58(2008), 2351-2379.

[47] T. Yang, A Batalin-Vilkovisky algebra suructure on the Hochschild cohomology of truncated polynomials, preprint (2007). 